\title{
Observational Evidence for the Coevolution of Galaxy Mergers, Quasars, and the Blue/Red Galaxy Transition
}

\section{Citation}

Hopkins, Philip F., Kevin Bundy, Lars Hernquist, and Richard S. Ellis. 2007. “Observational Evidence for the Coevolution of Galaxy Mergers, Quasars, and the Blue/Red Galaxy Transition." The Astrophysical Journal 659 (2): 976-96. https://doi.org/10.1086/512091.

\section{Permanent link}

http://nrs.harvard.edu/urn-3:HUL.InstRepos:41381599

\section{Terms of Use}

This article was downloaded from Harvard University's DASH repository, and is made available under the terms and conditions applicable to Other Posted Material, as set forth at http:// nrs.harvard.edu/urn-3:HUL.InstRepos:dash.current.terms-of-use\#LAA

\section{Share Your Story}

The Harvard community has made this article openly available. Please share how this access benefits you. Submit a story.

Accessibility 


\title{
OBSERVATIONAL EVIDENCE FOR THE COEVOLUTION OF GALAXY MERGERS, QUASARS, AND THE BLUE/RED GALAXY TRANSITION
}

\author{
Philip F. Hopkins, ${ }^{1}$ Kevin Bundy, ${ }^{2}$ Lars Hernquist, ${ }^{1}$ and Richard S. Ellis ${ }^{2}$ \\ Received 2006 May 22; accepted 2006 December 14
}

\begin{abstract}
We compile a number of observations to estimate the time-averaged rate of formation or buildup of red sequence galaxies, as a function of mass and redshift. Comparing this with the mass functions of mergers and quasar hosts, and independently comparing their clustering properties as a function of redshift, we find that these populations trace the same mass distribution, with similar evolution, at redshifts $0<z \lesssim 1.5$. Knowing one of the quasar, merger, or elliptical mass/luminosity functions, it is possible to predict the others. Allowing for greater model dependence, we compare the rate of early-type buildup with the implied merger and quasar triggering rates as a function of mass and redshift and find agreement. Over this redshift range, observed merger fractions can account for the entire bright quasar luminosity function and buildup of the red sequence at all but the highest masses at low redshift $\left(\gtrsim 10^{11} M_{\odot}\right.$ at $\left.z \lesssim 0.3\right)$ where "dry" mergers appear to dominate. This supports a necessary prediction of theories where mergers between gas-rich galaxies produce ellipticals with an associated phase of quasar activity, after which the remnant becomes red. These populations trace a similar characteristic transition mass, possibly reflecting the mass above which the elliptical population is mostly ( $\gtrsim 50 \%$ ) assembled at a given redshift, which increases with redshift over the observed range in a manner consistent with suggestions that cosmic downsizing may apply to red galaxy assembly as well as star formation. These mass distributions as a function of redshift do not uniformly trace the all/red/blue galaxy population, ruling out models in which quasar activity is generically associated with star formation or is long lived in "old" systems.
\end{abstract}

Subject headings: cosmology: theory — galaxies: active — galaxies: evolution — quasars: general

Online material: color figures

\section{INTRODUCTION}

Observations motivate the notion of "cosmic downsizing" (as coined by Cowie et al. 1996), with the global star formation rate (SFR) declining rapidly below $z \sim 2$, and the sites of galactic star formation shifting to smaller masses at lower redshift. Moreover, galaxy surveys such as SDSS, COMBO-17, and DEEP2 demonstrate that the color distribution of galaxies is bimodal (e.g., Strateva et al. 2001; Balogh et al. 2004) and that this bimodality extends at least to $z \sim 1$ (e.g., Bell et al. 2004; Faber et al. 2005).

It is increasingly established that high-mass, red elliptical galaxies have older stellar populations than smaller spheroids (e.g., Caldwell et al. 2003; Nelan et al. 2005; Gallazzi et al. 2006). However, many studies also see a significant population of massive/luminous galaxies in place (i.e., assembled) by $z \sim 2$ (e.g., Papovich et al. 2006; Renzini 2006 and references therein), with measurements of galaxy stellar mass functions (MFs) and luminosity functions (LFs) at redshifts $0<z<2$ favoring either a uniform increase or buildup in the numbers of early-type (red sequence [RS]) galaxies (e.g., Bell et al. 2004; Faber et al. 2005) or an antihierarchical scenario in which this buildup at $z \lesssim 1$ occurs primarily at the low-mass end of the RS (Bundy et al. 2005, 2006; Zucca et al. 2006; Yamada et al. 2005; Borch et al. 2006; Franceschini et al. 2006; Pannella et al. 2006; Cimatti et al. 2006; Brown et al. 2007). The blue, disk-dominated, star-forming galaxy MF (dominant at low mass), meanwhile, remains relatively constant, or perhaps declines to $z=0$. As a consequence, the transition mass, above which the red galaxy population dominates the galaxy MF, decreases with time, tracing this downsizing trend.

\footnotetext{
${ }^{1}$ Harvard-Smithsonian Center for Astrophysics, Cambridge, MA 02138.

2 California Institute of Technology, Pasadena, CA 91125.
}

There is evidence for some evolution at the highest masses as ellipticals grow by spheroid-spheroid or "dry" mergers (van Dokkum 2005; Bell et al. 2006b), but this, by definition, proceeds strictly hierarchically and cannot account for the movement of mass onto the RS in the first place or any buildup in the number density of low-mass ellipticals.

Meanwhile, the discovery of tight correlations between the masses of central supermassive black holes (BHs) in galaxies and the bulge or spheroid stellar mass (Magorrian et al. 1998), velocity dispersion (Gebhardt et al. 2000; Ferrarese \& Merritt 2000), or concentration (Graham et al. 2001) implies that the formation of galaxies and BHs must be linked. Moreover, the evolution of the quasar luminosity function (QLF) shows a sharp decline after $z \sim 2$, with the density of lower luminosity active galactic nuclei (AGNs) peaking at low redshift (e.g., Hasinger et al. 2005 and references therein). To the extent that BH assembly traces galaxy assembly (i.e., there is weak evolution in the BH-host mass relation, as observed to at least $z \gtrsim 1$ by, e.g., Shields et al. 2003; Adelberger \& Steidel 2005a; Peng et al. $2006)$, this implies early assembly times $(z \gtrsim 1)$ for many of the most massive systems containing $M_{\mathrm{BH}} \gtrsim 10^{8} M_{\odot}$ BHs.

A number of theoretical models have been proposed to explain the evolution of these populations with redshift and their correlations with one another (e.g., Kauffmann \& Haehnelt 2000; Somerville et al. 2001; Wyithe \& Loeb 2003; Granato et al. 2004; Scannapieco et al. 2005; Baugh et al. 2005; Monaco \& Fontanot 2005; Croton et al. 2006; Hopkins et al. 2006b, 2006c, 2006e; Cattaneo et al. 2006). In many of these models, the merger hypothesis (Toomre 1977) provides a potential physical mechanism linking galaxy star formation, morphology, and $\mathrm{BH}$ evolution and explaining these various manifestations of cosmic downsizing. In this scenario, gas-rich galaxy mergers channel large amounts of gas to galaxy centers (e.g., Barnes \& Hernquist 1991, 1996), 
fueling powerful starbursts (e.g., Mihos \& Hernquist 1994, 1996) and buried BH growth (e.g., Sanders et al. 1988; Barnes \& Hernquist 1992) until the BH grows large enough that feedback from accretion rapidly unbinds and heats the surrounding gas (Silk \& Rees 1998), leaving an elliptical galaxy satisfying observed correlations between $\mathrm{BH}$ and spheroid mass. Major mergers rapidly and efficiently exhaust the cold gas reservoirs of the progenitor systems, allowing the remnant to rapidly redden with a low specific SFR, with the process potentially accelerated by the expulsion of remnant gas by the quasar (e.g., Springel et al. 2005a). This naturally explains the observed close association between the elliptical and red galaxy populations (e.g., Kauffmann et al. 2003).

In a qualitative sense, the evolution of the characteristic mass at which these processes occur can be understood as follows. Mergers proceed efficiently at high redshift, occurring most rapidly in the regions of highest overdensity corresponding to the most massive galaxies, building up the high-mass elliptical MF. However, once formed these galaxies are "dead," and mergers involving gas-rich galaxies must transition to lower masses.

Recent hydrodynamical simulations, incorporating star formation, supernova feedback, and $\mathrm{BH}$ growth and feedback (Springel et al. 2005b), make it possible to study these processes self-consistently and have lent support to this general picture. Mergers with $\mathrm{BH}$ feedback yield remnants resembling observed ellipticals in their correlations with BH properties (Di Matteo et al. 2005), scaling relations (Robertson et al. 2006a), colors (Springel et al. 2005a), and morphological and kinematic properties (Cox et al. 2006a, 2006b). The quasar activity excited through such mergers can account for the QLF and a wide range of quasar properties at a number of frequencies (Hopkins et al. 2005d, 2006b), and with such a detailed model to map between merger, quasar, and remnant galaxy populations it is possible to show that the buildup and statistics of the quasar and red galaxy populations are consistent and can be used to predict one another (Hopkins et al. 2006c).

However, it is by no means clear whether this is, in fact, the dominant mechanism in the buildup of early-type populations and quasars and their evolution with redshift. For example, many semianalytic models incorporate quasar triggering/feedback and morphological transformation by mergers (Kauffmann \& Haehnelt 2000; Volonteri et al. 2003, 2006; Wyithe \& Loeb 2003; Somerville et al. 2004b; Monaco \& Fontanot 2005; Bower et al. 2006; Lapi et al. 2006; Menci et al. 2006). However, some models tie quasar activity directly to star formation (e.g., Granato et al. 2004), implying that it will evolve in a manner tracing starforming galaxies, with this evolution and the corresponding downsizing effect roughly independent of mergers and morphological galaxy segregation at redshifts $z \lesssim 2$. Others invoke poststarburst AGN feedback to suppress star formation on long timescales and at relatively low accretion rates through, e.g., radio-mode feedback (Croton et al. 2006), which, if this is also associated with optical QSO modes, would imply that quasars should trace the established "old" red galaxy population at each redshift. There are, of course, other sources of feedback, with galactic superwinds from star formation presenting an alternative means to suppress subsequent star formation, although the required wind energetics are sufficiently high to prefer a quasardriven origin (e.g., Benson et al. 2003). Several models invoke a distinction between hot and cold accretion modes (Birnboim \& Dekel 2003; Kereš et al. 2005; Dekel \& Birnboim 2006), in which new gas cannot cool into a galactic disk above a critical dark matter halo mass, potentially supplemented by AGN feedback (Binney 2004), as the dominant distinction between the blue cloud and RS, essentially independent of effects on scales within galaxies.

It is also important to distinguish the processes that may be associated with the initial movement of galaxies onto the RS from their subsequent evolution. Once morphologically transformed by a gas-rich merger, for example, mass can be moved up the RS (galaxies increased in mass) by gas-poor mergers, but it cannot be added to the RS in this manner. It also remains an important cosmological question to understand how, once formed, further growth of ellipticals by accretion or cooling flows may be halted. The models above invoke various feedback processes, including radio-mode activity (e.g., Croton et al. 2006), cyclic quasar or starburst-driven feedback (Somerville et al. 2001; Granato et al. 2004; Binney 2004; Monaco \& Fontanot 2005), massive entropy injection from a single quasar epoch (e.g., Wyithe \& Loeb 2003; Scannapieco \& Oh 2004), and hot mode accretion (Birnboim \& Dekel 2003), to address this problem. Although critical to our understanding of galaxy formation, these processes must operate over timescales of order the Hubble time for all massive galaxies once formed and therefore are not necessarily associated with the addition of mass to the RS. As such, the details of these long-term suppression mechanisms should be studied in different (e.g., already formed elliptical) populations and are outside the scope of this paper.

Observationally, it is still unclear whether mergers can account for the buildup of elliptical and/or quasar populations (see, e.g., Floyd et al. 2004; Rothberg \& Joseph 2006; Lotz et al. 2006b and references therein). Even within the context of the merger hypothesis, the relative importance of dissipational (gas-rich, disk) versus dissipationless (gas-poor, spheroid-spheroid) mergers is unclear (e.g., van Dokkum 2005; Bell et al. 2006b), although all measurements agree that the dry merger rate is much less than the gas-rich merger rate at all observed redshifts (Bell et al. 2006a, 2006b; Lotz et al. 2006b). This is essentially related to the critical question of whether the buildup of the RS and elliptical populations is dominated by the formation or movement of "new" early-type galaxies onto that sequence or instead by the hierarchical assembly of small "seed" early-type galaxies and substructure formed at high redshift (which will also not trigger quasar activity).

Fundamentally, it is not clear and has not yet been tested whether the observed downsizing trends in the transition mass, galaxy stellar populations, quasars, and other populations are in fact quantitatively the same trend, or merely qualitatively similar. This represents a key test that can distinguish between several of the various scenarios above. Attempting to predict the values of this transition mass in an a priori cosmological manner is inherently model dependent and, at least at low redshift, degenerate between the various models described above. However, if mergers are indeed the critical link in the process causing the flow of galaxies from the blue to red sequence and triggering quasar activity, then it is a strong prediction of these theories, and specifically the modeling of Hopkins et al. (2005a, 2005b, 2005c, 2005d, 2006a, 2006b, 2006c, 2006d), that the same mergers are responsible for the bulk of the bright quasar population and the buildup of the new mass on the RS at each redshift. In other words, these downsizing trends must quantitatively reflect one another.

In this picture, the transition mass $\left(M_{\mathrm{tr}}\right)$ may represent the "smoking gun" of mergers causing the flow of galaxies from the blue to red sequence. Therefore, to the extent that $M_{\text {tr }}$ traces the mass at which the RS is being built at some $z$, it should also trace the characteristic mass of star-forming galaxies merging at that time and the characteristic mass of galaxies hosting quasars that are initially triggered by those mergers. Of particular interest, 


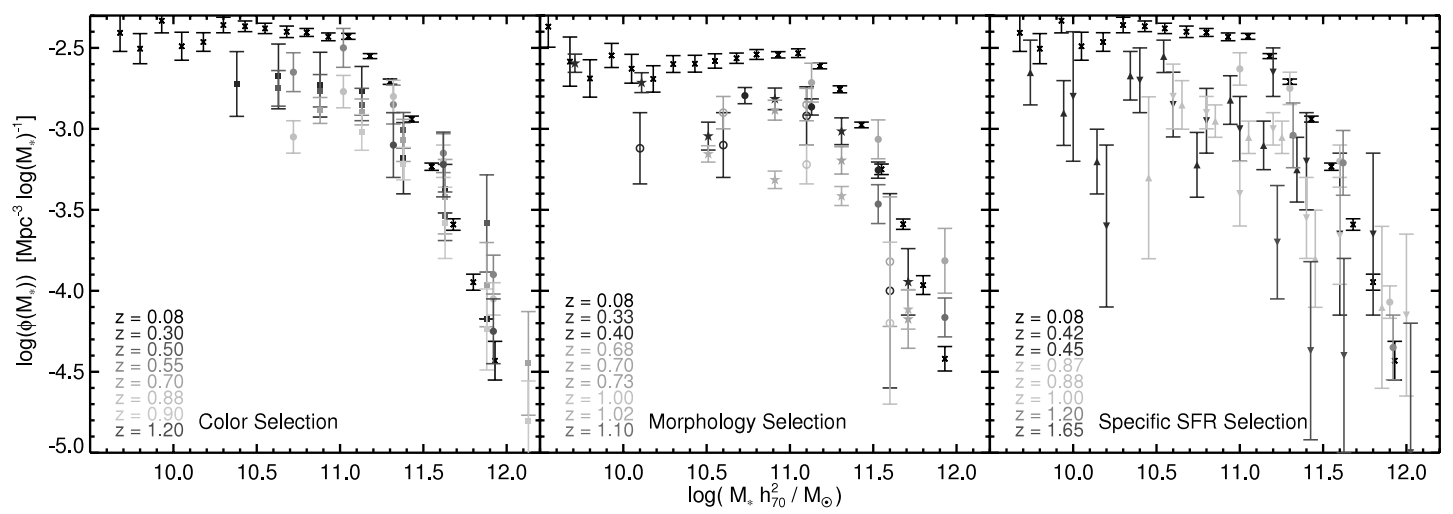

Fig. 1.-MFs of early-type galaxies selected by color, morphology, or specific SFR, from Bell et al. (2003; crosses), Borch et al. (2006; squares), Bundy et al. (2005, 2006; filled circles), Pannella et al. (2006; open circles), Franceschini et al. (2006; stars), Fontana et al. (2004; downward-pointing triangles), and Pozzetti et al. (2003; upward-pointing triangles). Points are shaded by redshift, as labeled, and shown only above the quoted completeness limits of each study. The data have been converted to our adopted cosmology and masses rescaled to a Salpeter (1955) IMF. Comparison suggests that cosmic downsizing may apply in some sense to early-type galaxy assembly, as well as star formation histories. [See the electronic edition of the Journal for a color version of this figure.]

the empirical test of this association does not require the adoption of some a priori model for galaxy formation.

Here we consider the observed $M_{\text {tr }}$ over the interval $0<z<2$ and compare it to the characteristic masses of quasar hosts and merging galaxies over the same range in redshift. We demonstrate that they appear to be evolving in a manner consistent with a merger-driven unification model of quasars, interacting galaxies, and the red galaxy population. Note that we use the term "quasar" somewhat loosely, as a proxy for high Eddington ratio accretion inevitably caused by gas-rich mergers, although there may be other triggering mechanisms as well (Sanders et al. 1988; Alexander et al. 2005a, 2005b; Borys et al. 2005; Hopkins et al. 2006 b). Such activity will of course be significantly weaker in small systems (especially those typical of local ultraluminous infrared galaxies [ULIRGs]) and may not technically qualify as a "classical" optical quasar (Hopkins et al. 2005a), but this distinction is essentially arbitrary and has little impact on our analysis (see also Hopkins et al. 2006e).

We adopt an $\Omega_{M}=0.3, \Omega_{\Lambda}=0.7, H_{0}=70 \mathrm{~km} \mathrm{~s}^{-1} \mathrm{Mpc}^{-1}$ cosmology. All stellar masses are rescaled to a Salpeter (1955) initial mass function (IMF).

\section{THE TRANSITION MASS AND BUILDUP OF EARLY-TYPE POPULATIONS}

\subsection{Defining the Transition Mass}

Various studies have used different definitions and terms for the mass that separates the dominance of old, red, low-SFR elliptical galaxies from that of young, blue, star-forming disk galaxies. It is also possible to divide the galaxy population along any one of those quantities. Although it has been established in a number of observational studies that the galaxy population is bimodal with respect to color, specific SFR, and morphology (e.g., Strateva et al. 2001; Kauffmann et al. 2003; Balogh et al. 2004; Driver et al. 2005) and that this bimodality extends at least to $z \sim 1$ (e.g., Bell et al. 2004; Faber et al. 2005), it is still possible that the various definitions used to separate these bimodal distributions could result in a systematically different "separation point."

In what follows, we consider several definitions of the transition galaxy stellar mass in terms of the MFs $[\phi(M)]$ of early- and late-type systems: the Bundy et al. (2005) transition mass $M_{\mathrm{tr}}$ at which the density of early- and late-type systems are equal,

$$
\phi_{\text {early }}\left(M_{\mathrm{tr}}, z\right)=\phi_{\text {late }}\left(M_{\mathrm{tr}}, z\right),
$$

the Bundy et al. (2006) quenching mass $M_{Q}$ at which the contribution of late types to the total MF cuts off,

$$
\phi_{\text {late }}(M, z)=\phi_{\text {all }}(M, z) \exp \left(-M / M_{Q}\right)
$$

and the Cimatti et al. (2006) downsizing mass $M_{50}$ above which $50 \%$ of the $z=0$ RS MF has been assembled by a given redshift,

$$
\phi_{\text {early }}\left(M>M_{50}, z\right) \geq 0.5 \phi_{\text {early }}\left(M>M_{50}, z=0\right) .
$$

For each, we consider a division between early and late types defined by either color (i.e., separating galaxies on the redshiftdependent RS from the "blue cloud;" see, e.g., Bell et al. 2004), SFR (generally from detailed population synthesis modeling, although we include looser emission/absorption galaxy spectral type separations), or morphology (either by visual morphological identification or concentration/surface brightness) criteria. Above these masses, early-type galaxies in the red, low-SFR, high-concentration, morphologically elliptical half of the bimodal distribution dominate the total galaxy MF, and below, late-type galaxies in the blue, high-SFR, low-concentration, morphologically disklike or irregular bimodal half dominate (generally true also for $M_{50}$, although it is technically independent of $\phi_{\text {late }}$ ).

We determine these masses from a number of compiled typeseparated MFs, shown in Figure 1 (for clarity, just the early-type MFs are shown). Data in all cases are converted to our adopted cosmology and rescaled to a Salpeter (1955) IMF. At all redshifts, only points above the quoted completeness limits of each study are shown. Errors are as published and generally account for cosmic variance similar to, e.g., Somerville et al. (2004a). At $z=0$, we generally adopt the local MF determinations from Bell et al. (2003) from 2MASS+SDSS observations but find no change in our results considering, e.g., the $2 \mathrm{MASS}+2 \mathrm{dFGRS}$ Cole et al. (2001) determination. At higher redshifts, our compilation includes Pozzetti et al. (2003; K20, $z<1.3$ ), Fontana et al. (2004; K20, $z<2.0$ ), Bundy et al. (2005; GOODS, $z<1.4$ ) and Bundy et al. (2006; DEEP2, $z<1.4$ ), Borch et al. (2006; COMBO-17, $z \leq 1.0$ ), Franceschini et al. (2006; CDF-S, $z<1.4$ ), and Pannella et al. (2006; FORS Deep+GOODS-S, $z \leq 1.15$ ). Each of these considers the separate MFs of early and late types, divided according to at least one of the criteria above. Although 


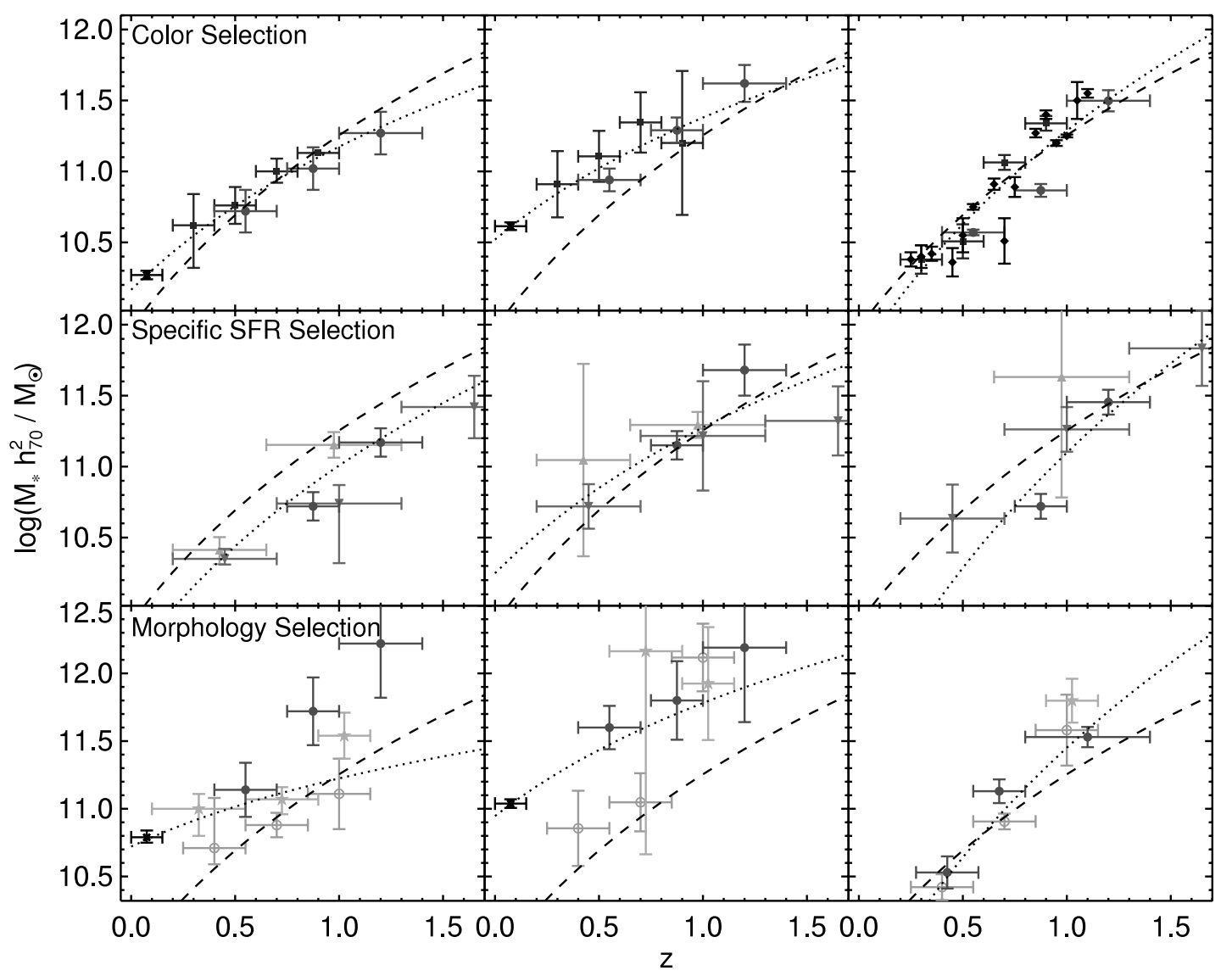

FIG. 2.-Evolution with redshift of the transition or downsizing mass, at which objects may be in transition from the blue cloud to the RS (i.e., the characteristic mass at which elliptical populations may be building up) with different sample selections and definitions of this mass. Left: $M_{\mathrm{tr}}$, the mass above which ellipticals dominate the cumulative galaxy MF, with galaxy types separated by color selection (top), specific SFR or spectral (absorption/emission) fitting (middle), and morphological selection (bottom). Middle: $M_{Q}$, the mass at which the contribution of late-type galaxies cuts off, with the same sample definitions as for $M_{\mathrm{tr}}$. Right: $M_{50}$, the mass above which the early-type MF at $z$ is $\geq 50 \%$ assembled relative to the Bell et al. (2003) MF at $z=0$ [i.e., $\phi\left(M>M_{50}, z\right) \geq 0.5 \phi\left(M>M_{50}, z=0\right)$ ]. Data are shown from the MFs in Fig. 1, in the same point style, shaded by the observed sample: Bell et al. (2003; crosses), Borch et al. (2006; squares), Bundy et al. (2005, 2006; filled circles), Pannella et al. (2006; open circles), Franceschini et al. (2006; stars), Fontana et al. (2004; downward-pointing triangles), and Pozzetti et al. (2003; upward-pointing triangles). We also consider $M_{50}$ calculated in Cimatti et al. (2006; diamonds) from the LFs of Bell et al. (2004), Willmer et al. (2006), Faber et al. (2005), and Yamada et al. (2005) using the redshift-dependent mass-to-light ratios estimated from fundamental plane studies. Dotted lines in each panel show the best-fit trend of the form $M_{*} \propto(1+z)^{k}$. Dashed lines (identical in all panels) show a cumulative best fit to the $M_{50}$ data from all samples. Although there are systematic factor of $\sim 2$ normalization offsets between different methods that caution against mixing definitions, the various methods all trace a similar mass. Regardless of the characteristic mass definition, the sample survey, or the method of type segregation of the samples, a similar trend with redshift is recovered in each case. [See the electronic edition of the Journal for a color version of this figure.]

many of these surveys cover a small area and thus may be subject to significant cosmic variance, our conclusions are unchanged if we restrict ourselves to the largest fields and are in fact most robust for the wide-field surveys from COMBO-17 $\left(0.78 \mathrm{deg}^{2}\right)$ and DEEP2 (3.5 $\left.\mathrm{deg}^{2}\right)$.

Figure 2 shows resulting transition mass from these studies as a function of redshift, from the different definitions and galaxy type segregation methods above. These samples generally derive masses from optical+near-IR spectral and photometric fitting. To compare, we also consider the evolution in $M_{50}$ (color selected) determined by Cimatti et al. (2006). They compile the $B$-band early-type (red) galaxy LFs from COMBO-17 (Bell et al. 2004), DEEP2 (Willmer et al. 2006; Faber et al. 2005), and the Subaru $X M M$-Newton Deep Survey (Yamada et al. 2005) at $z=0-1.15$ (typical $\Delta z \approx 0.05$ between LFs) and use the redshift-dependent evolution of $B$-band mass-to-light ratios determined from fundamental plane studies (e.g., van Dokkum \& Stanford 2003; van der Wel et al. 2005; Treu et al. 2005; di Serego Alighieri et al. 2005; Renzini 2006; roughly similar to mean formation redshifts $z_{f} \sim 3-4$ ) to correct these to a $z=0$ equivalent $B$ luminosity and mass (given the $z=0$ massdependent $B / g$-band $M / L$ ratios from Bell et al. 2003). We fit a simple relation of the form

$$
M_{*} \propto(1+z)^{\kappa}
$$

in each panel (for illustrative purposes only; we do not intend for this to be considered a rigorous estimate of the evolution in these characteristic masses).

Despite the different selection and type separation methods and definitions of a characteristic mass, a nearly identical trend with redshift is recovered in every case. In fact, the best-fit slopes $\kappa$ for most selection methods and definitions are statistically indistinguishable from the cumulative best-fit slope, and several of the definitions agree nearly exactly in Figure 2 . It is also reassuring that the indirect estimates from optical LFs, which generally involve the largest samples and most finely probe the redshift evolution of $M_{50}$, agree well at all redshifts with the MF estimates. This suggests that the trend with redshift is real and that it is independent of the potential systematics in sample 
selection, as these systematics can be quite different for the various criteria shown.

The absolute normalization of the transition mass does depend systematically on the definition chosen. For example, same separation by color or SFR gives a systematic factor of $\sim 2$ lower mass than separation by morphology, and $M_{Q}$ is systematically higher than $M_{\mathrm{tr}}$ by about the same factor for all separation methods (see also Bundy et al. 2006). The systematic difference in $M_{\text {tr }}$ and $M_{Q}$ can be understood as a consequence of their definitions (essentially one could define arbitrary Schechter functions for early- and late-type systems, and as long as the early-type function has a shallower slope and larger $M_{*}$, this small systematic offset in the two measurements would be guaranteed). The systematic offset between color/SFR and morphological selection is best demonstrated in the detailed comparison of local color and morphologically selected MFs in, e.g., Bell et al. (2003). These authors find that although the different selection methods preserve the same qualitative behavior and result in early- and late-type samples that are identical in $~ 80 \%-90 \%$ of the included galaxies, the color criterion does result in a slightly larger number of early-type systems (probably owing to the large scatter in blue galaxy colors, with a nonnegligible highly dust-reddened population), which will push the characteristic separation mass slightly lower. This may also explain why there appears to be a larger scatter between samples at a given redshift in the morphologically defined $M_{\text {tr }}$ and $M_{Q}$, as such an effect will be sensitive to a given sample's resolution and imaging depth. There may also be an interesting timescale effect, as discussed in Bundy et al. (2006) if galaxies redden onto the RS somewhat more rapidly than they morphologically relax following mergers (perhaps suggesting different mechanisms for morphological and color transformation). However, that $M_{50}(z)$ (depending only on $\left.\phi_{\text {early }}\right)$ is similar regardless of selection method suggests that these differences may be an artifact of the selection/identification of blue galaxies. In any case, further detailed study of these intermediate objects and comparison between different samples is needed to understand these differences.

These systematic distinctions caution against mixing definitions in determining the redshift evolution of these masses. However, for our purposes, the systematic normalization scatter of a factor of $\sim 2$ is not large: this is comparable to the inherent ambiguity in defining a characteristic mass of any population (e.g., Schechter $M_{*}$ ). As long as we are careful about the relatively small normalization offsets between selection criteria, we can safely compare the transition mass and its evolution with redshift to the masses of other populations.

\subsection{The Buildup of Ellipticals and the Physical Significance of the Transition Mass}

It has been suggested (e.g., Bundy et al. 2005) that the transition mass may represent the mass at which the early-type MF is building up at each redshift, in the sense that new spheroids are being added to the RS MF at this mass. If cosmic downsizing applies to galaxy assembly to any extent, i.e., this building up extends at higher masses at higher redshift (or peaks at lower redshift for lower masses), then this implies a shift of $M_{50}$ to lower mass at lower redshift (Cimatti et al. 2006). To the extent that the shape of the late-type MF does not dramatically change over this redshift interval (observed in the samples above and, e.g., Feulner et al. 2003; Drory et al. 2004) this will, by definition, manifest in a similar evolution of $M_{\mathrm{tr}}$ and $M_{Q}$. In this scenario, then, $M_{50}$ is directly tied to downsizing in the earlytype MF.
Figure 3 considers the evolution with redshift of the number density of early-type galaxies of a given $M_{*}$, from $9.75 \leq$ $\log \left(M_{*} / M_{\odot}\right) \leq 12.5$. At low $M_{*}$, the number density declines steeply with $z$ [roughly $\propto(1+z)^{-\beta}$ with $\beta=2.37$ ]. Although the statistics are poor and variance large at high $M_{*}$, there is a significant trend $(\sim 7 \sigma)$ for a shallower decline in number density at higher masses ( $\beta \sim 0$, i.e., little evolution in number density, at $\left.M_{*} \gtrsim 10^{12} M_{\odot}\right)$. Above $z \sim 1$, the various samples plotted begin to disagree, and the $\propto(1+z)^{-\beta}$ functional form may not be a good approximation, so we reconsider this, fitting only the data at $z \leq 1.0(z \leq 0.5)$, and find the same trend at $\sim 6 \sigma(\sim 4 \sigma)$.

Figure 4 considers the differential growth of the early-type MF in more detail. We show the time-averaged buildup of earlytype MFs in several redshift intervals from $z=0$ to 1.5 . Where a given sample measures the elliptical MF at two redshifts $z$ and $z+\Delta z$, we differentiate the observed elliptical MF at every mass with respect to the two redshifts to obtain the time-averaged rate of creation of ellipticals of that mass, over that redshift interval. In total, we show four redshift intervals: $z \sim 0.0-0.3,0.3-0.7$, $0.7-1.0$, and 1.0-1.5. Of course, we only compare MFs measured with the same technique and sample in the same study, since systematic offsets in methodology could severely bias such an estimate. Although the scatter is large (especially at low masses), the observations all trace a similar elliptical formation rate as a function of mass, with a similar break traced in the different samples at each redshift. The shape of this function is not the same as that of the early-type MFs; i.e., we are not simply recovering the fact that the MF builds up uniformly over these intervals.

At low redshift $(z \lesssim 0.3)$, comparison with the early-type MFs in Figure 1 shows that sub- $M_{*}\left(M \lesssim 10^{11} M_{\odot}\right)$ ellipticals are building up in number density by $\sim 7 \%-15 \% \mathrm{Gyr}^{-1}$, whereas the most massive systems build up by only $\sim 1 \% \mathrm{Gyr}^{-1}$. In other words, the most massive systems are not building up (via either star formation or assembly of stellar populations) at a significant rate at low redshift. At high $z$, there is a marginal shift of this function to higher masses. By $z \sim 1$, comparison with the corresponding early-type MFs implies that systems with $M_{*} \gtrsim$ $10^{11} M_{\odot}$ are building up by $\sim 20 \%-50 \% \mathrm{Gyr}^{-1}$. The best-fit Schechter functions plotted in each redshift interval reflect this, with the Schechter $M_{*}$ shifting from $11.02 \pm 0.11(z \lesssim 0.3)$ to $11.51 \pm 0.07(1.0 \lesssim z \lesssim 1.5)$.

The low- $z$ growth estimate of $\sim 1 \% \mathrm{Gyr}^{-1}$ in the most massive systems is in excellent agreement with that from Masjedi et al. (2006) and Bell et al. (2006a) determined from local red galaxy (spheroid-spheroid or dry) merger rates. In detail, observations suggest that the typical massive red galaxy undergoes $\sim 0.5-1$ major dissipationless mergers since $z=1$ (van Dokkum 2005; Bell et al. 2006b). If this is representative, it is trivial to predict the corresponding rate of buildup of the elliptical population, assuming that every red galaxy undergoes this number of major ( $\sim 1: 1$ mass ratio) mergers in this time. This is shown in Figure 4, calculated from the local MF of Bell et al. (2003). Although these mergers appear to be important for building up the most massive galaxies at low $(z \lesssim 0.3)$ redshift, their contribution cuts off completely below $\sim 2 \times 10^{11} M_{\odot}$ (and will cut off at higher masses at higher $z$, generally $\sim 2$ times the break in the buildup MF). Such mergers move galaxies from the low-mass end of the elliptical MF to the high-mass end, and since the low-mass slope of the elliptical MF is not steep, this can only decrease the number density of lowmass objects. By definition, then, dry mergers cannot account for the (substantial) buildup of total mass on the RS or the buildup at low and intermediate masses. If these lower mass ellipticals are formed by gas-rich mergers, then there must be at least $\sim 2$ times 


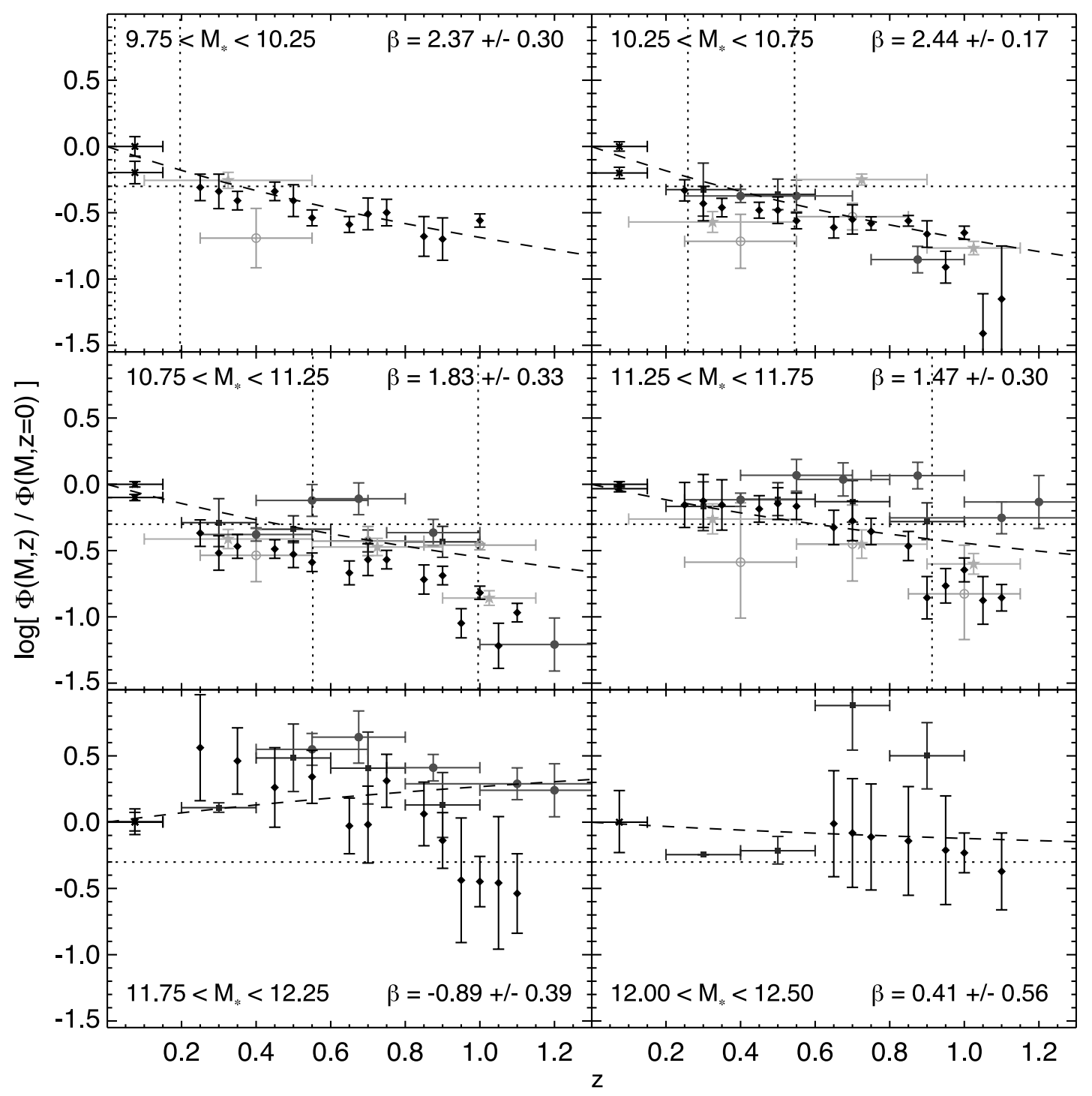

FIG. 3.-Number density of early-type galaxies in different mass bins as a function of redshift, relative to that at $z=0$ from Bell et al. (2003; color selected) from the samples in Fig. 2 (same style). The dotted horizontal line shows $50 \%$ of the $z=0$ value, while dotted vertical lines show the redshifts at which the lower and upper limits of each mass bin correspond to the best-fit $M_{50}(z)$ (dashed line in Fig. 2). Dashed lines in each panel show the best-fit trend of the form $\propto(1+z)^{-\beta}$, with the labeled $\beta$. Although there is considerable variance at high masses, a mass-independent galaxy density evolution $\beta$ can be ruled out at $\sim 7 \sigma$. A steeper $\beta$ at low mass implies that a large fraction of these galaxies are added at lower redshift than galaxies of higher mass. [See the electronic edition of the Journal for a color version of this figure.]

as many gas-rich mergers moving new galaxies onto the elliptical MF as there are dry mergers (in fact, the actual observed ratio is $\sim 10$ : 1; Bell et al. 2006b).

\section{THE TRANSITION MASS AND CHARACTERISTIC GALAXY MASSES}

Figure 5 compares the transition mass determined above with the characteristic masses (Schechter $M_{*}$ ) of the red, blue, and all galaxy populations. For clarity, we show just $M_{50}$, as it is the most well determined of the masses in Figure 2, as well as being most robust with respect to sample definitions/selection, and further it has the most direct physical interpretation (as it is not degenerate with blue cloud evolution). Our conclusions here and subsequently, however, are unchanged regardless of the mass definition from Figure 2. The characteristic masses of red, blue, and all galaxies are nearly constant with redshift, with at most a marginal ( $\sim 0.2$ dex) increase from $z=0$ to 1 , inconsistent with their following the strong trend seen in $M_{50}$ at $\gtrsim 10 \sigma$ (independent of normalization). Likewise, comparing the shape of the rate of elliptical buildup in Figure 4 with these galaxy MFs at the same redshift shows that they do not trace the same mass distribution as a function of redshift. We can therefore (perhaps unsurprisingly, given the definitions employed) rule out at high significance the hypothesis that transition mass objects are uniformly/randomly drawn from a parent population of normal galaxies of either early or late (or both) types.

It may appear that the strong trend in $M_{50}$ (i.e., the mass above which the RS MF is $>50 \%$ assembled at $z$ ) is incompatible with the weak trend in $M_{*}$ of red galaxies (as, e.g., no change in $M_{*}$ would imply uniform buildup of RS populations at all masses). This, however, is an artifact of the Schechter function fit. For example, given a local early-type Schechter function MF with $\phi_{*}=\phi_{0}, \alpha$, and $M_{*}=M_{0}$ and a similar MF at $z$ with $\phi_{*}=\phi_{z}$, the same $\alpha$ (commonly assumed in fitting), and $M_{*}=M_{0}(1+\delta)$, one obtains

$$
M_{50}(z)=M_{0}\left(1-\frac{1}{1+\delta}\right)^{-1} \ln \left[\frac{\phi_{0}}{2 \phi_{z}}(1+\delta)^{\alpha+1}\right],
$$




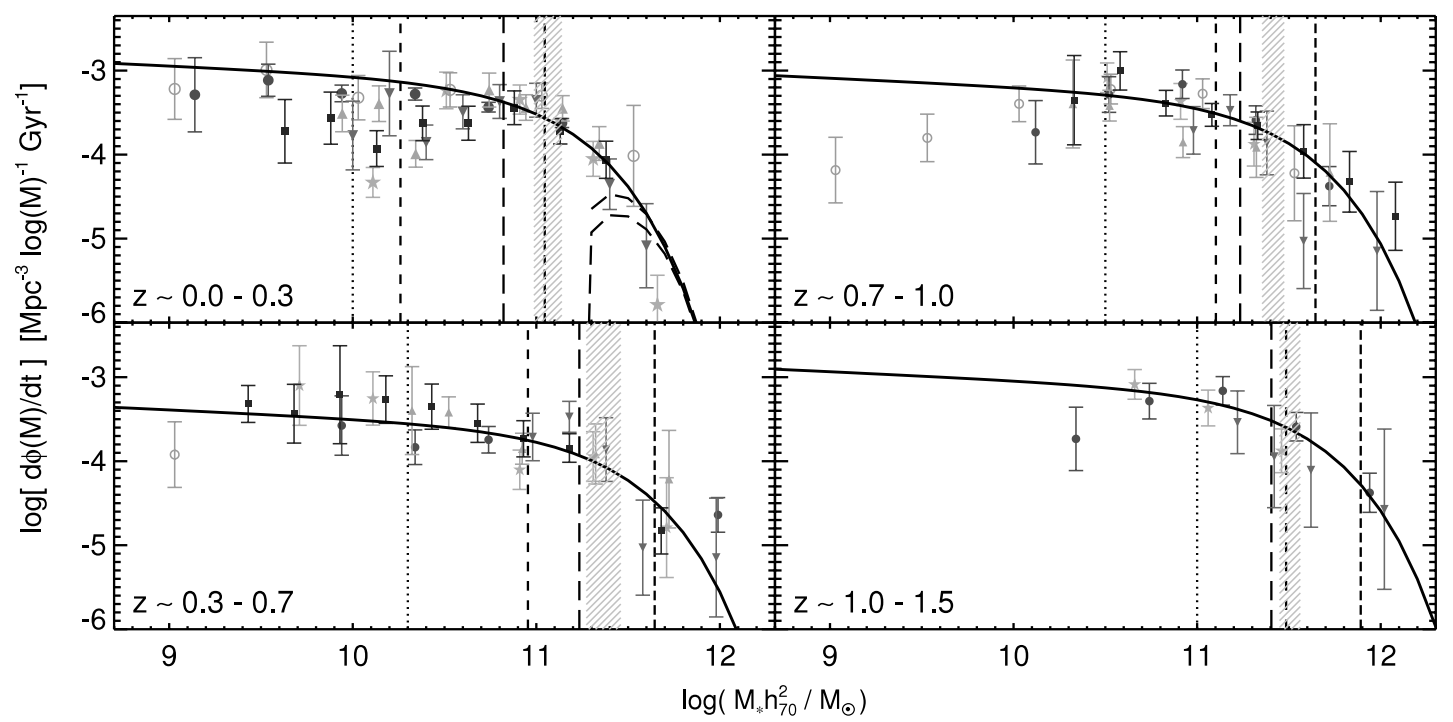

FIG. 4. - Time-averaged rate of formation of elliptical galaxies, obtained by differentiating the observed elliptical MFs in Fig. 1 with respect to time ( points; style as in Fig. 2). Results are shown over a number of redshift intervals, as labeled. Dotted lines show typical completeness limits at each redshift. Short-dashed, long-dashed, and dot-dashed vertical lines show $M_{50}, M_{\mathrm{tr}}$, and $M_{Q}$ from Fig. 2 (color-selected fits) at each $z$. The solid line in each panel shows the best-fit Schechter function (to points above the completeness limits quoted for each sample), with the shaded range showing the $1 \sigma$ range of the best-fit $M_{*}$. High-mass ellipticals appear to preferentially build up at higher redshifts. The characteristic masses in Fig. 2 provide a reasonable proxy for the characteristic $M_{*}$ being added or built up in the early-type galaxy MF at each redshift, as both evolve to higher masses at higher redshift. In the top panels, thick dashed lines show the maximal contribution from spheroid-spheroid mergers if all undergo $\sim 0.5-1$ (lower and upper lines) such mergers in that redshift interval (at or below the break at each $z$, a significant dissipationless merging fraction will lower the number density at low $M$, where it is observed to rise). [See the electronic edition of the Journal for a color version of this figure.]

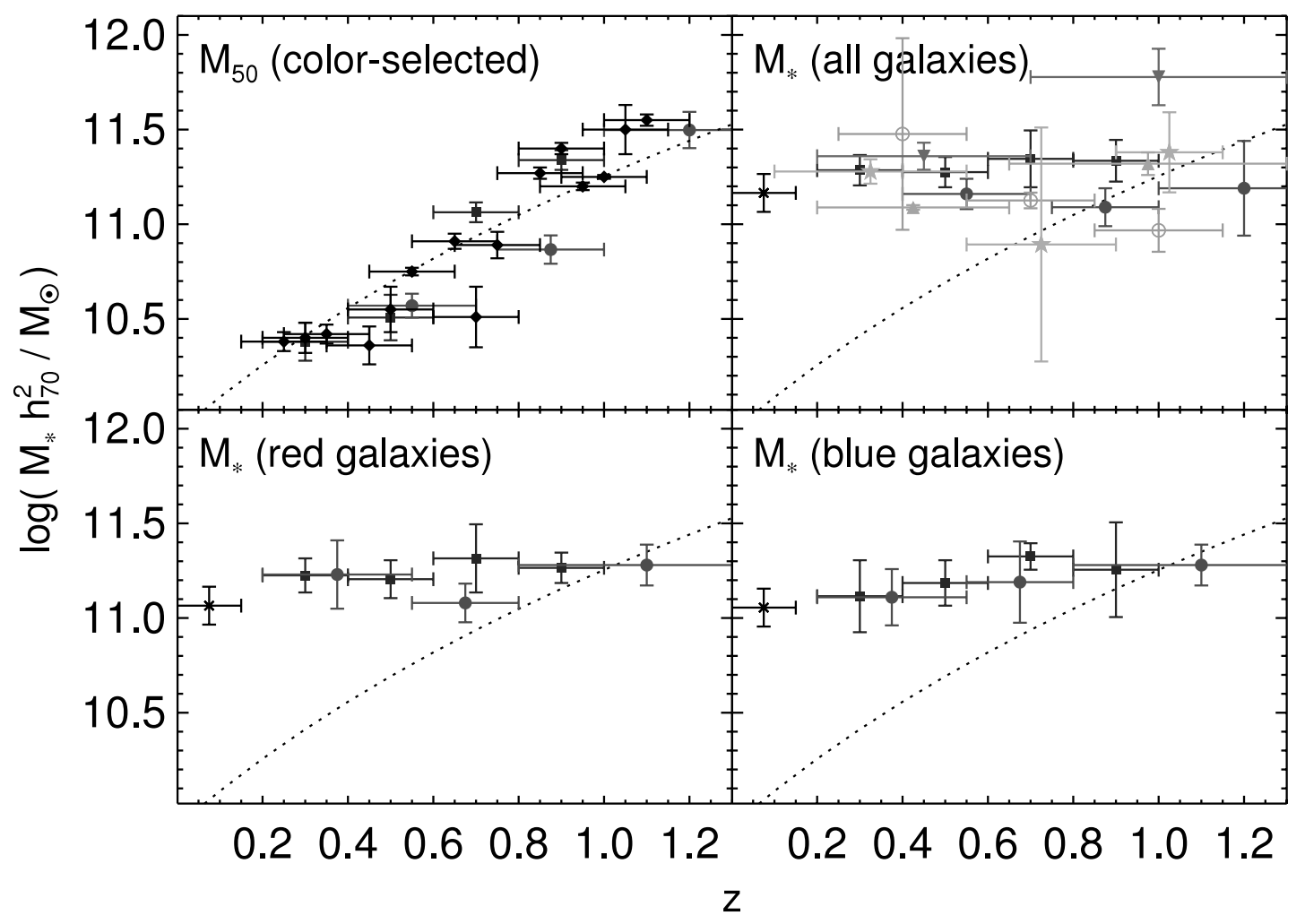

Fig. 5.-Comparison of the downsizing or transition mass with characteristic galaxy masses. Top left: $M_{50}$ (points; from color-selected samples) and best-fit trend (dotted line) from Fig. 2. Other panels show the best-fit Schechter function $M_{*}$ to the all galaxy MFs and color-selected red and blue galaxy MFs (points as in Fig. 2). The trend in $M_{50}$ (and $M_{\mathrm{tr}}, M_{Q}$ ) does not trace the all, blue, or red galaxy populations; i.e., transition mass systems are not uniformly drawn from any of these populations. Note that the trend in $M_{50}$ is not incompatible with that in $M_{*}$ of red galaxies, as the Schechter function parameterization is such that an order-of-magnitude change in $M_{50}$ at a constant rate of elliptical formation implies only a $\sim 0.1-0.2$ dex change in $M_{*}$ (which can be further offset by a small dry merger rate). [See the electronic edition of the Journal for a color version of this figure.] 

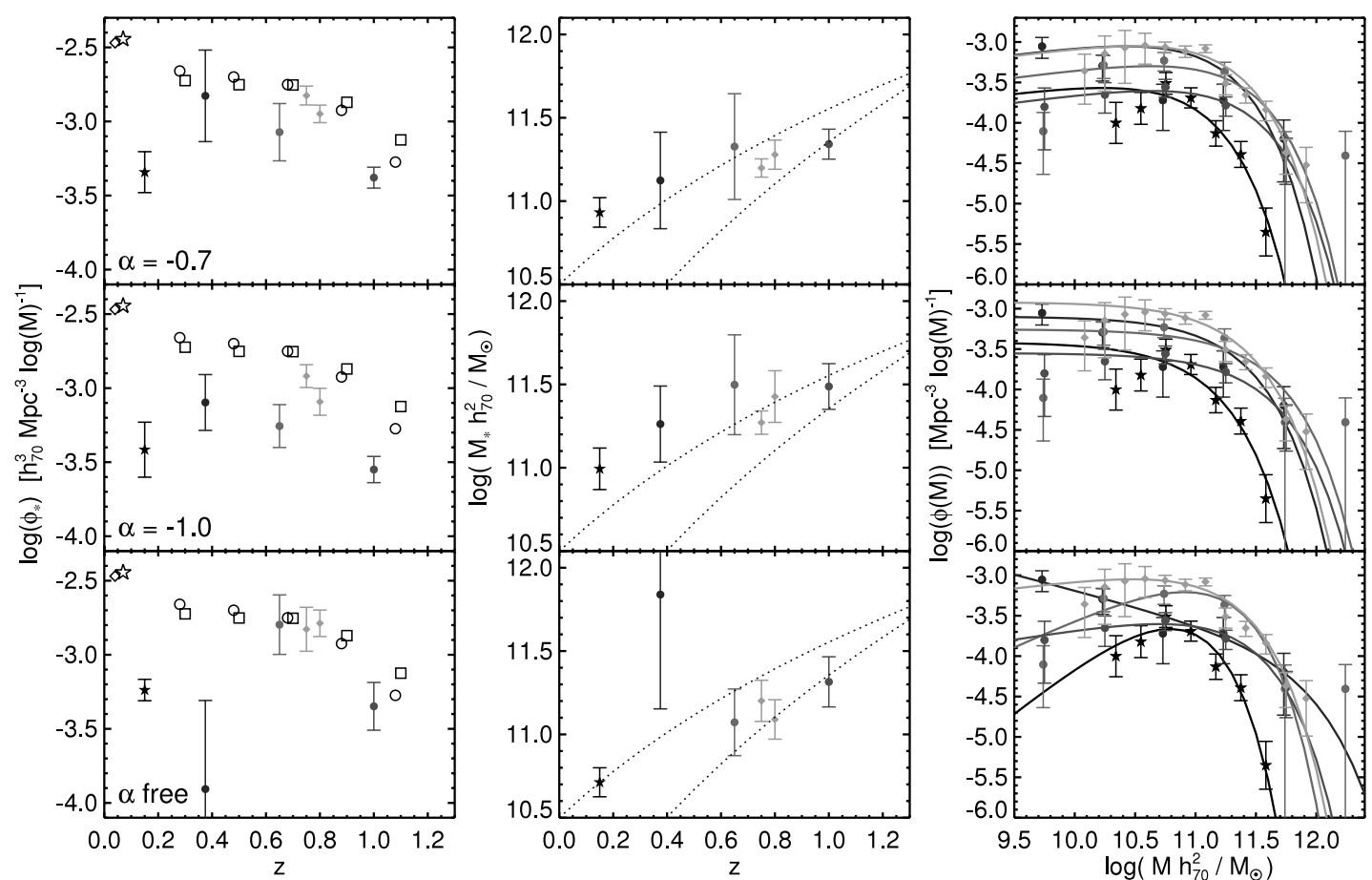

FIG. 6.-Comparison of observed merger MFs from Xu et al. (2004; stars) at $z \lesssim 0.2$, Bundy et al. $(2005,2006 ;$ circles $)$ at $z=0.2-0.5, z=0.5-0.8$, and $z=0.8-1.2$, and Wolf et al. (2005; diamonds) at $z=0.7-1.0$ (upper and lower $z$ points from GEMS and GOODS, respectively). Panels show the merger MFs (right), with best-fit Schechter functions (lines of corresponding shade), and best-fit Schechter function $\phi_{*}($ left $)$ and $M_{*}$ (middle). Because constraints on the faint-end slope are weak, top panels fix its value $\alpha=-0.7$, middle panels fix $\alpha=-1.0$, and bottom panels allow $\alpha$ to be fitted. Open points in the left panels show $\phi_{*}$ of the entire galaxy population as compiled in Faber et al. (2005) from Bell et al. (2003; SDSS; star), Madgwick et al. (2003; 2dF; diamond), Bell et al. (2004; COMBO-17; circles), and Willmer et al. (2006; DEEP2; squares). Dotted lines in the middle panels show the fitted $M_{50}(z)$ (lower) and $M_{\mathrm{tr}}(z)$ from Fig. 2 (color selected). Regardless of the choice of $\alpha$, there is a trend for the characteristic merger mass $M_{*}$ to increase with redshift in a manner similar to $M_{\mathrm{tr}}$. Interpretation of $\phi_{*}$ is more ambiguous, but there is a suggestion that it increases relative to the $\phi_{*}$ of the galaxy population. [See the electronic edition of the Journal for a color version of this figure.]

which is quite sensitive to $\delta$ and, for the observed values of $\phi_{*}(z), M_{*}(z)$ (e.g., Borch et al. 2006), predicts a $\sim 1-2$ dex evolution in $M_{50}$ similar to what we find.

In a more physical sense, the local RS MF is the sum of the RS MFs built up over various intervals, each of which resembles a Schechter function (see Fig. 4). Consider the sum of two Schechter functions with identical $\alpha$ and $\phi_{*}$, but one having lower $M_{*}$ by $1 \mathrm{dex}$. Fitting this to a Schechter function over the range $M_{*} \pm 1 \mathrm{dex}\left( \pm 1.5 \mathrm{dex}\right.$ ) yields a best fit with $M_{*}$ only lowered from the higher value by $\sim 0.1 \operatorname{dex}(0.05 \mathrm{dex})$. This owes to the steep fall in $\phi(M)$ at $M \gg M_{*}$, which forces the combined fit to retain the high $M_{*}$ value. In further detail, if we imagine that the rate of buildup $d \phi(M) / d t(z)$ (Fig. 4) is a Schechter function with constant $\alpha$ and normalization, but an evolving $M_{*}=$ $M_{50}(z)$ [adopting the best-fit trend $M_{50}(z)$ shown in Fig. 5], and then fit the integrated $\phi_{\text {early }}(M, z)$ to a Schechter function (fitting over the range $M_{*} \pm 1$ dex), we obtain only $\sim 0.15-0.20$ dex evolution in the early-type $M_{*}$ from $z=0$ to 1 , despite the more than order-of-magnitude evolution in $M_{50}(z)$. Thus, although the strong evolution in the transition mass with redshift rules out its being representative of the general elliptical population, it is not inconsistent with the weak evolution in the early-type $M_{*}$, even if $M_{50}$ does represent the characteristic mass at which new galaxies are being added to the RS. In other words, weak evolution in $M_{*}$ of red galaxies does not rule out strong evolution in the characteristic masses being built up on or added to the RS.

\section{THE TRANSITION MASS AND MERGERS}

We next consider observed merger MFs. We compile the local $(z \leq 0.2)$ pair-selected major (within $\sim 1 \mathrm{mag}$ ) merger LFs from Xu et al. (2004; 2MASS) in $K$ band and Toledo et al.
(1999) in $B$ band, as well as the morphologically identified merger/interacting galaxy LFs from Brinchmann et al. (1998; CFRS+LDSS, $z \leq 1$ ) in $B$ band and Wolf et al. (2005; GEMS+ GOODS, $z \sim 0.7)$ in the near-UV $(280 \mathrm{~nm})$, and MFs from Conselice et al. (2003, 2005; HDF-N and HDF-S, $z \sim 1-3)$ and Bundy et al. (2006; GOODS+DEEP2, $z \leq 1.4$ ). Where the MFs are not directly measured, we rescale the LFs to MFs using the mass-to-light ratios of ongoing mergers (as a function of $M_{*}$ ) from Hopkins et al. (2006e; see their Table 1). These are calculated from the population synthesis models of Bruzual \& Charlot (2003) given the distribution of star formation histories during mergers determined from several hundred numerical simulations that include star formation, supernova feedback and metal enrichment, and $\mathrm{BH}$ accretion and feedback (for details see Hopkins et al. 2006d; this is essentially a second-order improvement on the typical empirically adopted simplified tau+burst models for these $M / L$ ). These should be reasonably robust: they have also been checked directly in the bands of interest here against the measurements of $M / L$ ratios in local ULIRGs (Tacconi et al. 2002), pair samples (Dasyra et al. 2006), and recent merger remnants (Rothberg \& Joseph 2004) and give good agreement (Hopkins et al. 2006e, their Figs. 1 and 4). Furthermore, Hopkins et al. (2006e, their Figs. 8 and 9) demonstrate that they can be reliably used to convert merger LFs to MFs (in exactly this manner) for all samples above where both are measured. In any case, our subsequent results are unchanged (albeit their significance reduced given the limited data) if we consider only the morphologically identified, directly measured merger MFs of Conselice et al. $(2003,2005)$ and Bundy et al. (2006).

Figure 6 shows several (the most well constrained) of these MFs as a function of redshift, with the best-fit Schechter functions. 


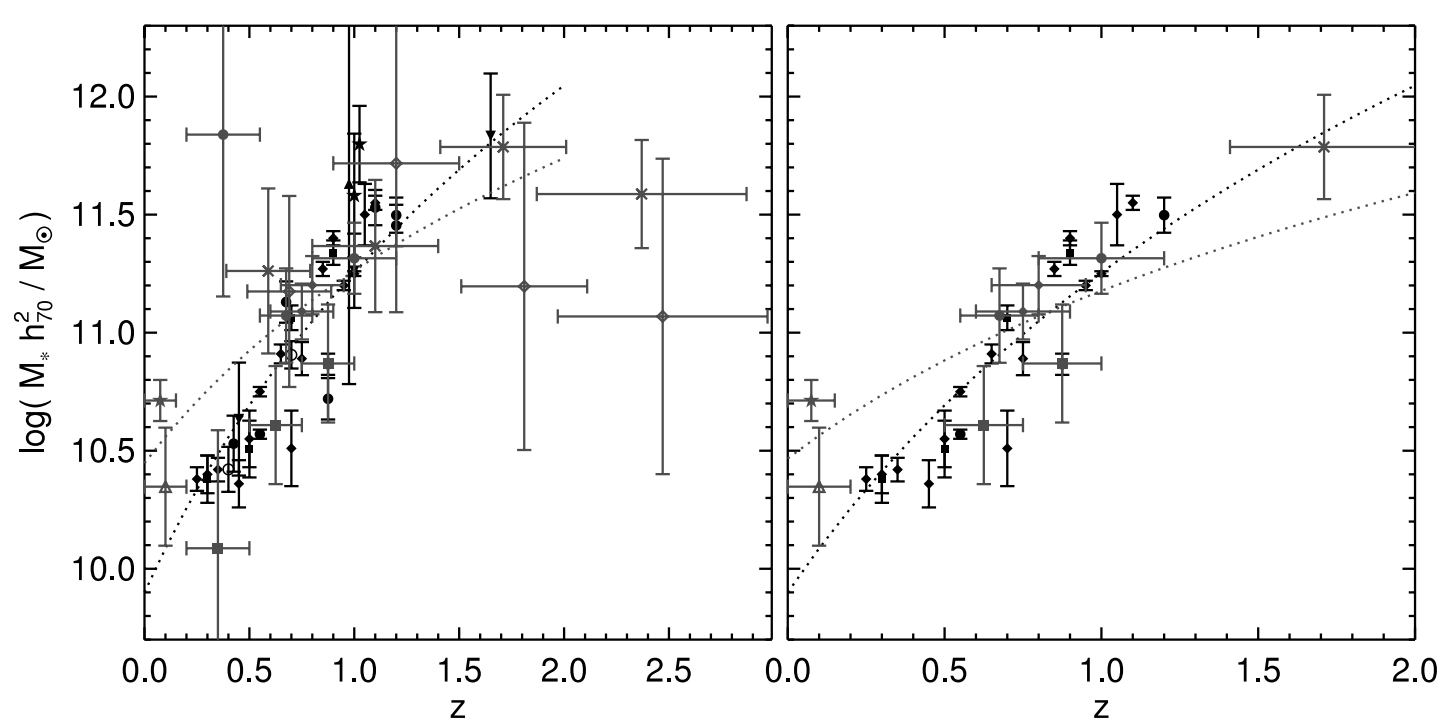

FIG. 7. - Left: Transition mass $M_{50}$ from all sample selections shown in Fig. 2 (black points in same style), and the best-fit trend with redshift (black dotted line), compared to the characteristic mass $M_{*}$ from merger MFs and LFs ( gray points; see Fig. 6), from Xu et al. (2004; stars), Wolf et al. (2005; filled diamonds), Bundy et al. (2005, 2006; circles), Conselice et al. (2003; HDF-S, crosses; HDF-N, open diamonds), Toledo et al. (1999; triangles), and Brinchmann et al. (1998; squares) with the best-fit trend of the form $M_{*} \propto(1+z)^{\kappa}$ (gray dotted line). The $M_{*}$ values shown allow the merger MF faint-end slope $\alpha$ to vary freely, but a similar result is obtained fixing $\alpha$ to match the early-type or all galaxy values (see Fig. 6). Right: Same as the left panel, but for clarity, only $M_{50}$ from color-selected samples and the bestconstrained $\frac{1}{2}$ of merger $M_{*}$ values are shown. Despite the small sample sizes, the characteristic mass of merger MFs increases with redshift at $>3 \sigma$ (implying that mergers are not simply drawn from the approximately constant $M_{*}$ all, blue, or red galaxy populations) and is consistent with the value and evolution of $M_{50}$ as a function of redshift, as in Fig. 8. [See the electronic edition of the Journal for a color version of this figure.]

Since constraints are weak at the faint end (and systematic uncertainties large; discussed below), we consider fits with fixed faint-end slopes $\alpha$, as well as allowing $\alpha$ to vary. In all cases, there is a qualitatively similar trend for the Schechter function $M_{*}$ to increase ( by $\sim 0.5-0.7 \mathrm{dex}$ from $z=0$ to 1 ). Direct comparison of the MFs demonstrates that this does not necessarily mean that the number of mergers at the high-mass end increases monotonically with redshift (changes in $\phi_{*}$ can offset the increase in $M_{*}$ ); this does, however, imply that the relative merger history/MFs favor higher mass mergers at higher redshifts. Although it is not important for our comparison, there is also a marginal trend for $\phi_{*}$ of mergers to increase with redshift relative to $\phi_{*}$ of the entire galaxy population, but this trend is largely driven by the low $\phi_{*}$ from $\mathrm{Xu}$ et al. (2004).

Figure 7 plots these Schechter $M_{*}$ values, as well as those determined from the other merger mass/luminosity functions we compile, as a function of redshift, compared to the characteristic transition mass $\left(M_{50}\right)$. The characteristic masses from pair and morphologically selected samples, as well as direct MFs and optical, near-IR, and near-UV LFs, agree surprisingly well at overlapping redshifts, at least up to the $z \gtrsim 2$ estimates that are strongly affected by cosmic variance (Conselice et al. 2005), which further suggests that the typical merger $M / L$ ratios used are reasonable. There is a significant $(>3 \sigma)$ trend for the characteristic masses of mergers to increase with redshift. As with the transition mass population in Figure 5, this trend rules out at $>3 \sigma$ the hypothesis that mergers are randomly/uniformly drawn from the all or red galaxy population. Whether mergers uniformly trace the blue galaxy population is less clear; the values plotted are inconsistent with this hypothesis at $\sim 3.5 \sigma$, but the trend alone (i.e., allowing for a systematic normalization offset) is inconsistent at only $\sim 2 \sigma$. The values/trend of the merger MF $M_{*}$ as a function of redshift are, however, similar and statistically consistent with $M_{50}$ (and even more similar to $M_{\text {tr }}$ and $M_{Q}$; see Fig. 17 below).

We can consider in greater detail if observed merger mass/ luminosity functions are consistent with the observed buildup of early-type populations by examining the complete MFs as a function of redshift. Figure 8 reproduces Figure 4 but overlays the observed merger MFs from Figure 6 at the appropriate observed redshifts. Since there is considerable ambiguity in converting an observed merger MF to a merger rate, we renormalize (vertically only, i.e., divide out an appropriate timescale) the observed MFs arbitrarily such that we can focus here just on the unambiguous mass distribution (although we will consider the issue of absolute rates/normalization shortly). The agreement is striking: independent of the systematics in understanding merger rates, the observed distribution of mergers as a function of mass and redshift traces and is consistent with the buildup/addition of galaxies to the RS at all masses and redshifts observed.

Although systematically uncertain, we should also compare the implied merger rates (i.e., vertical normalization in Fig. 8). In other words, even if the buildup of early-type populations traces the merger mass distribution, are there the appropriate total number/rate of mergers to account for the growth of the RS MF (assuming that mergers are the agent of this buildup)? To estimate this, we assume that every elliptical formation/addition event in Figure 4 indeed owes to a merger, which is observable as such for some amount of time $t_{\text {merger }}$ (until morphological disturbances such as tidal tails fade beyond typical surface brightness limits). This yields the expected merger MF, $\phi\left(M_{\text {merger }}\right) \sim t_{\text {merger }} \dot{\phi}\left(M_{\text {gal }}\right)$. Given the galaxy MFs from which we calculated these rates in the first place, it is then trivial to estimate the expected merger fraction as a function of mass.

Figure 9 compares this estimate, adopting a characteristic $t_{\text {merger }}=0.5 \mathrm{Gyr}$, with observed merger fractions as a function of mass and redshift. This timescale is roughly expected from numerical simulations of mergers (e.g., Robertson et al. 2006b), dynamical friction considerations (Patton et al. 2002), or more detailed estimates of observational selection effects as a function of merger stage (for a detailed discussion of these issues see, e.g., Hopkins et al. 2006e). We consider the merger fraction above two representative stellar mass limits, $M_{*}>10^{10} M_{\odot}$ 


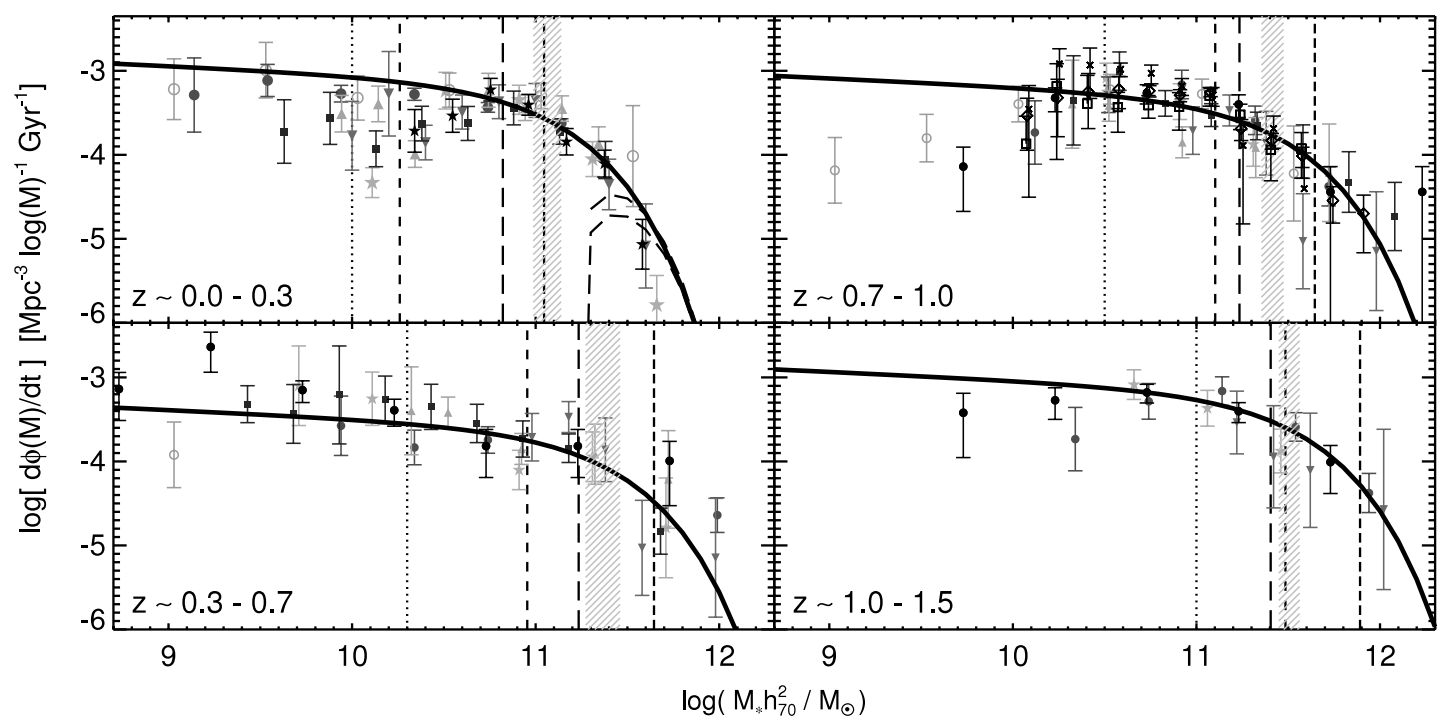

FIG. 8. - Time-averaged rate of buildup of early-type populations, as in Fig. 4, but with the observed merger MFs from Fig. 6 overlaid (black points; style as in Fig. 6). Merger MF points at $z \sim 0.7-1.0$ from Wolf et al. (2005) show the effects of systematic differences in imaging depth and survey area, from GEMS (squares), GOODS (crosses), and GEMS+GOODS (diamonds). Since the merger timescale is observationally undetermined, we compare the mass distribution in mergers and the early-type buildup (i.e., merger MFs are renormalized for direct comparison). The time-averaged buildup of elliptical populations traces a similar mass distribution to that of observed merger populations at all redshifts observed, and not the same mass distribution as that of the all, red, or blue galaxy population as a function of redshift (compare Fig. 5). [See the electronic edition of the Journal for a color version of this figure.]

and $M_{*}>10^{11} M_{\odot}$, but caution that these are not, for the most part, mass-selected samples, so the mass limits shown in Figure 9 are only broadly applicable. We calculate the merger fraction as a function of mass directly from the merger MFs of Xu et al. (2004), Wolf et al. (2005), and Bundy et al. (2005) shown in Figure 6, at redshifts where the samples are complete to the given mass limit (this essentially excludes the $z \gtrsim 1$ Bundy et al. [2005] merger MF).
The observed merger fractions are consistent with this estimate at all redshifts. The buildup of elliptical populations does suggest that merger fractions should increase as a function of redshift, but we note that the effect is quite weak. If, for example, the characteristic merger timescale decreases with redshift in the same manner as halo dynamical times (at fixed mass), $\propto(1+z)^{-3 / 2}$ (decreasing the expected merger fraction we calculate by this amount), the expected increase in merger fraction with redshift

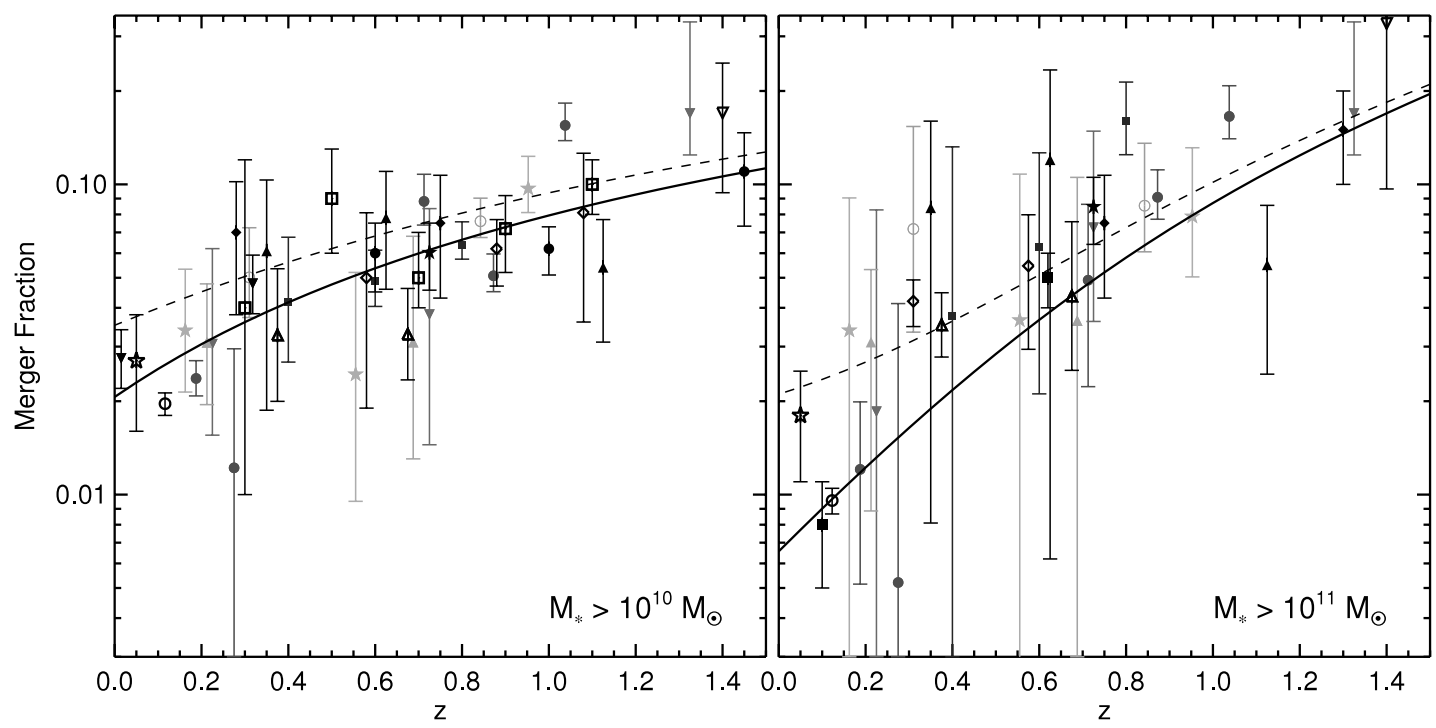

FIG. 9.-Expected merger fraction as a function of redshift, if the buildup of the early-type MFs in Fig. 6 is entirely a result of mergers moving galaxies onto the RS (shaded points; style as in Fig. 6). Black points show observed merger fractions as a function of redshift, from Patton et al. (2002; filled downward-pointing triangles), Conselice et al. (2003; filled circles), Bundy et al. (2004; filled upward-pointing triangles), Lin et al. (2004; open diamonds), Xu et al. (2004; open stars), De Propris et al. (2005; open circles), Cassata et al. (2005; filled diamonds), Wolf et al. (2005; filled stars), Bundy et al. (2005; open upward-pointing triangles), Lotz et al. (2006a; open downward-pointing triangles), Lotz et al. (2006b; open squares), and Bell et al. (2006a; filled squares). Results are shown for two (approximate) minimum stellar mass limits, as labeled. The solid line shows the expected gas-rich merger fraction if all bright quasars are triggered in mergers, using the same modeling from Fig. 12 to determine the quasar-parent MF from the observed QLF. The dashed line adds a constant fraction (observed 0.015; Bell et al. 2006b; Lotz et al. 2006b) of dissipationless (spheroid-spheroid) mergers. An observable merger timescale of $0.5 \mathrm{Gyr}$ is assumed. The expected merger fractions from the observed buildup of early-type MFs and the QLF agree reasonably in their normalization and evolution with observed merger fractions. There are sufficient mergers to account for both populations and little room for a large fraction of mergers that do not produce a remnant elliptical or trigger quasar activity. Dissipationless mergers are generally a relatively small effect, as is observed, but may be important for the buildup of the most massive systems at low $(z \lesssim 0.5)$ redshifts. [See the electronic edition of the Journal for a color version of this figure.] 
becomes marginal (factor $\lesssim 2$ by $z \sim 1.5$ ). Both cases, however, are consistent with the present observations (at these redshifts, within the factor $\sim 2$ typical uncertainties). There is also marginal evidence for steeper evolution in merger fractions with redshift in the higher mass cut we consider, evidence for which is also seen in, e.g., Conselice et al. (2003, 2005), but we caution both that this trend appears only when different samples are combined and that it will be "washed out" by the increasing importance of dry mergers at high masses and low redshifts (see also Fig. 13 below). Future studies that can separate gas-rich and gas-poor merger populations and track the merger fraction as a function of redshift and mass can provide a substantially stronger test of these trends.

Caution regarding systematic uncertainties in merger populations is still warranted. Le Fèvre et al. (2000) find that pair and morphological selection criteria yield similar results, but Lin et al. (2004) see significant disagreement, which may be the result of systematic identification of "false" (i.e., nonmerging) pairs at low redshift (Berrier et al. 2006). Fortunately, the characteristic merger mass (or luminosity) does not appear to change dramatically with selection method even though the time spent in a given phase (and thus $\phi_{*}$ or merger fraction) may. For example, the data of Lin et al. (2004) and Conselice et al. (2003) do yield a similar characteristic merging galaxy luminosity $L_{*}$, despite finding different merger fractions. One might also wonder whether the natural tendency of a flux-limited sample to select brighter systems at higher redshift might lead one to infer an increasing mass scale regardless of the underlying mass distribution. However, Figure 6 demonstrates that the completeness limits for most of the samples we consider are generally well below the transition mass and similarly below the break in the corresponding MF. Further, although Wolf et al. (2005) find that the observed number of faint mergers depends on selection effects (see Fig. 8, which shows the increase in number of lowmass mergers when increasing imaging depth and decreasing field size by an order of magnitude, from GEMS to GOODS), this does not significantly affect the merger $M_{*}$ or change our comparison in Figure 8. Still, these effects must be accounted for in any comparison of fitted MFs.

It is furthermore true that the exact appropriate value of the duration of observable merger activity $\left(t_{\text {merger }}\right)$ is not well determined and will in detail depend on the sample, mass limit, and redshift, but for our purposes these effects (amounting to a systematic factor $\sim 2$ uncertainty) are generally comparable to or smaller than the scatter in the observations in Figure 9. Preliminary estimates of the observable merger timescale based on comparison with automated nonparametric classification schemes (Lotz et al. 2004) suggest, perhaps surprisingly, relatively weak trends with redshift (at least at $z \lesssim 2$; see the discussion in Hopkins et al. 2006d), but lacking a complete cosmological framework from which to predict observable merger properties, Figure 9 should be taken with the strong caveat that the relative normalizations of galaxy buildup and observed mergers depend systematically on $t_{\text {merger }}$.

\section{THE TRANSITION MASS AND E+A GALAXIES}

Detailed studies of $\mathrm{E}+\mathrm{A}$ (or $\mathrm{K}+\mathrm{A}$ ) galaxies (Dressler \& Gunn 1983), with characteristic poststarburst stellar populations indicating a substantial but rapidly quenched star formation epoch in the last $\sim 0.1-0.5$ Gyr (Caldwell et al. 1996; Couch \& Sharples 1987; Quintero et al. 2004), have found ubiquitous evidence of morphological disturbances and tidal tails (Schweizer 1996; Blake et al. 2004; Goto 2005), which together with their environmental (e.g., Zabludoff et al. 1996; Goto 2005) and structural/

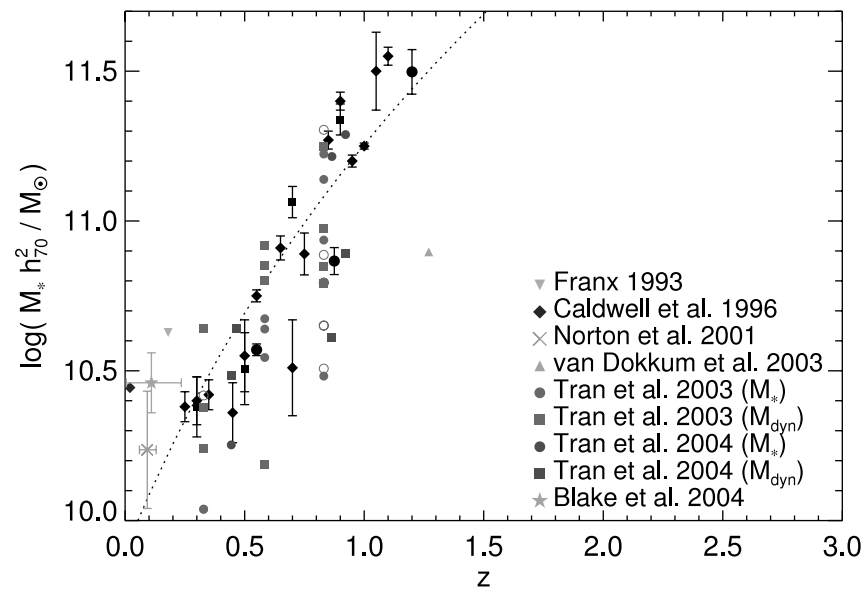

FIG. 10.- Observed mass of E+A galaxies (colored points, as labeled) compared to the transition mass $M_{50}$ (black points, as in Fig. 7, from color selection) as a function of redshift. We show the fitted $M_{*}$ of the E+A MF where available (points from Norton et al. [2001] and Blake et al. [2004] with error bars, as labeled), but owing to limited samples otherwise show the masses of individual $\mathrm{E}+\mathrm{A}$ galaxies observed in clusters (Franx 1993; Caldwell et al. 1996; van Dokkum \& Stanford 2003; both stellar and dynamical masses from Tran et al. [2003] are shown, as labeled) and in the field (Tran et al. 2004). The masses of E+A galaxies appear to trace the characteristic masses of mergers and the transition mass as a function of redshift, but lacking larger samples at $z>0$ from which to determine a full $\mathrm{E}+\mathrm{A} \mathrm{MF}$, it cannot be determined whether or not this is merely a selection effect. [See the electronic edition of the Journal for a color version of this figure.]

kinematic properties (Kelson et al. 2000; Norton et al. 2001; Tran et al. 2003; van der Wel et al. 2004) imply their formation in mergers and evolution into typical early-type galaxies. We therefore consider whether $\mathrm{E}+\mathrm{A}$ galaxies, presumably recently formed in mergers, trace any downsizing trend.

Figure 10 compares $M_{50}$ and the masses of observed E+A galaxies as a function of redshift. At low redshift, sizable samples exist, and we show the characteristic Schechter function $M_{*}$ of $\mathrm{E}+\mathrm{A}$ populations. At higher redshift, samples are extremely limited, and we can only plot the masses of individual systems. The points as plotted appear to downsize, as noted in Tran et al. (2003), but this trend could well be completely driven by survey flux limits. Lacking volume-limited samples or complete $\mathrm{E}+\mathrm{A}$ MFs at high redshift, we can only presently say that the $\mathrm{E}+\mathrm{A}$ data are not inconsistent with the downsizing in the transition mass or any of the other hypotheses considered herein.

\section{THE TRANSITION MASS AND QUASARS}

\subsection{The QLF Break Expected from the Transition Mass}

If the formation/movement of galaxies on the RS is associated with a quasar trigger (for example, through quasar feedback being an agent of reddening, or both being associated with a merger), then the observed QLF should reflect the rate of elliptical formation/buildup shown in Figure 4; indeed, in such a model, each quasar "broadcasts" a galaxy moving to/forming on the RS. (If the quasar lifetime were of order the Hubble time, of course, then the QLF would reflect the integrated/established early-type population, but observations constrain it to be much less at all redshifts [e.g., Martini 2004; Hopkins et al. 2006d], such that the QLF tracks the rate of triggering.)

It is straightforward to compare these. A spheroid of mass $M_{*}$ hosts a $\mathrm{BH}$ of mass $M_{\mathrm{BH}}=\mu M_{*}(\mu \approx 0.001$; Magorrian et al. 1998; Marconi \& Hunt 2003), confirmed by direct observations at all redshifts of interest $(z \lesssim 2$; Shields et al. 2003; Peng et al. 2006; Adelberger \& Steidel 2005a). A quasar event is essentially 
defined by ignition of the BH for a brief time $\left(t_{Q} \lesssim 10^{7} \mathrm{yr}\right.$ from various observations; see Martini 2004 and references therein) near the Eddington limit $L=3.3 \lambda \times 10^{4} L_{\odot}\left(M_{\mathrm{BH}} / M_{\odot}\right)$ (where $\lambda=L / L_{\text {Edd }} \approx 1$; e.g., McLure \& Dunlop 2004; Kollmeier et al. 2006). Thus, the formation or movement to the RS of a spheroid of mass $M_{*}$ would be associated, in this scenario, with a shortlived quasar of luminosity

$$
\frac{L_{\mathrm{bol}}}{L_{\odot}}=33\left(\frac{\lambda \mu}{0.001}\right) \frac{M_{\mathrm{host}}}{M_{\odot}} .
$$

This simple effective conversion for bright quasars is supported by both numerical simulations of quasars and galaxy mergers (Hopkins et al. 2006b) and direct comparison of quasar and host galaxy luminosities (Vanden Berk et al. 2006; Peng et al. 2006; Richards et al. 2006b). There is, of course, some uncertainty and observed scatter in the host galaxy-BH mass correlation and bright quasar Eddington ratios, but it is constrained to a factor of $\sim 2$, comparable to the uncertainty in the observed $M_{\text {tr }}$.

If this is the dominant mode of quasar triggering, then although the exact normalization of the QLF (number of observed quasars) will depend on the duty cycle $\delta$ and quasar lifetime $t_{Q}$, the break $M_{*}$ in the host/source MF (break in the rate of formation of early-type galaxies calculated in Fig. 4) will translate directly to a break $L_{*}$ (from eq. [6]) in the QLF. If $\delta$ and/or $t_{Q}$ are complicated functions of mass, luminosity, or redshift, they might change the slopes of the resulting QLF but will not move the break location $L_{*}$. Thus, $L_{*}$ directly tracks the characteristic mass of the host population. Put another way, essentially all observed $L \gtrsim L_{*}$ quasars have $\lambda \sim 1$, i.e., define a characteristic active BH mass $M_{\mathrm{BH}} \propto L_{*}$, and since $M_{*} \sim M_{\mathrm{BH}} / \mu$ at all redshifts of interest, the characteristic host mass $M_{*}$ of quasars is well defined at each $z$.

Figure 11 compares the QLF characteristic luminosity expected (eq. [6]) from objects of the transition mass $\left(M_{50}\right)$ and the observed characteristic QLF break luminosity $L_{*}$ as a function of redshift. The break $L_{*}$ is determined in the standard fashion, fitting the observed QLF at each redshift to a double power law. We show $L_{*}$ measured from optical, soft X-ray, and hard X-ray studies, each converted (to enable direct comparison) to a bolometric luminosity $L$ using a standard observationally derived bolometric correction (template quasar SED) and reddening correction (Hopkins et al. 2007 and references therein; note that adopting the less recent bolometric corrections from Elvis et al. [1994] or Marconi et al. [2004] yields nearly identical results). We also show the break determined by Hopkins et al. (2007; see their Table 2), who compile a large number of QLF measurements through the mid- and near-IR, optical, near-UV, soft and hard $\mathrm{X}$-ray, and soft gamma ray and use these to directly determine the bolometric QLF. In any case, the observed $L_{*}$ is robust; in fact, the (typical factor $\sim 2$ ) discrepancies in $L_{*}$ owe mostly to the data binning and fitting function, and a direct comparison of the data in Hopkins et al. (2007) shows that they trace a similar turnover/ break. We also note that the existence of a break is unambiguous (detected at $\gg 10 \sigma$ in most of the samples in Fig. 11), regardless of whether it is sharp (as expected for a double power-law fit) or exhibits some higher order curvature (as for a Schechter function; e.g., Wolf et al. 2003; Richards et al. 2005). It is unaffected by questions of completeness, as the X-ray surveys are typically complete to $\sim 2$ orders of magnitude in luminosity below $L_{*}$ (e.g., Hasinger et al. 2005). The break luminosity also increases with redshift (at least to $z \sim 2$ ), as has long been recognized in quasar surveys (recently, e.g., Boyle et al. 2000; Ueda et al. 2003; Croom et al. 2004; Richards et al. 2005; Hasinger et al. 2005;

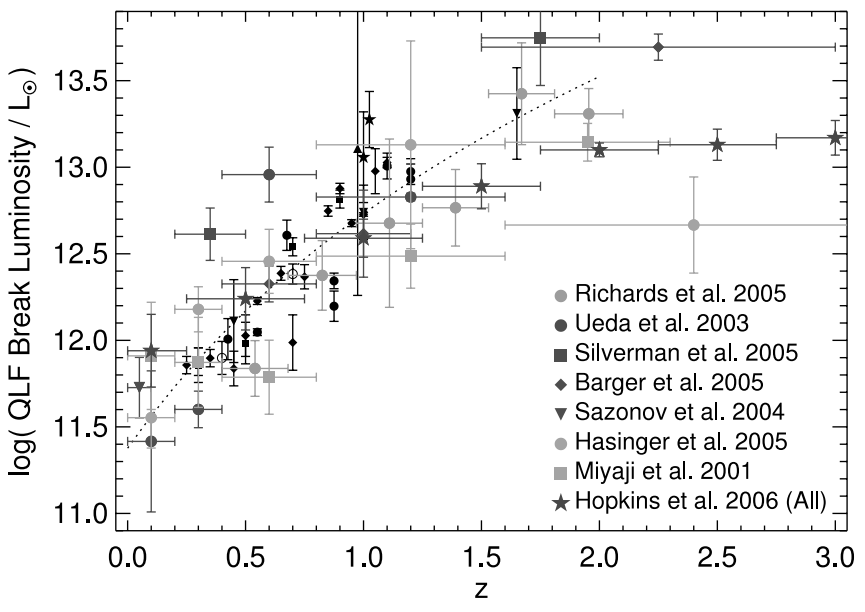

FIG. 11.-Predicted location of the QLF break $L_{*}$ as a function of redshift, from the observed transition mass (black points show $M_{50}$ as in Fig. 2, from all samples; dotted line shows best-fit trend), compared to the observed QLF break (shaded points) from various studies. The mass $M_{50}$ is converted to a luminosity assuming that the characteristic luminosity $L_{*}$ of quasars traces their host masses, since these objects are all observed to be near-Eddington and the BH-host mass relation evolves weakly with redshift to $z \lesssim 1$ (alternatively, convolving the mass distribution being added to early-type populations in Fig. 4 with some probability of seeing the $\mathrm{BH}$ in each system at a given luminosity extending to $\sim L_{\text {Edd }}$ yields a similar $L_{*}$ ). The QLF measurements shown from hard X-ray, soft X-ray, and optical are converted to bolometric luminosities with the observationally determined bolometric and dust corrections in Hopkins et al. (2007). The bolometric break luminosity directly fitted in Hopkins et al. (2007) from the compilation of the samples shown and $\sim 30$ other measured QLFs (see references therein) is also shown. The observed break luminosity from all samples is consistent with the expectation of any model in which the objects in Fig. 4 (objects being added or moving to the early-type population) are associated with quasar triggers, with the BHs appropriate for their stellar mass $M_{50}(z)$ briefly accreting near Eddington. [See the electronic edition of the Journal for a color version of this figure.]

significant in each case at $\gg 6 \sigma$ ), regardless of higher order subtleties implied by "luminosity-dependent density evolution" (e.g., Hasinger et al. 2005 and references therein) models and other changes in the detailed QLF shape as a function of redshift (e.g., Richards et al. 2006a; Hopkins et al. 2007).

That the expected $L_{*}$ from $M_{50}$ agrees with the observations is not surprising. Hopkins et al. (2006e) combined observed merger MFs with a large suite of corresponding hydrodynamical merger simulations (Robertson et al. 2006a), including BH accretion and feedback, to calculate what the resulting mergerdriven QLF should be in each band at each corresponding redshift. Their predicted merger-driven QLF agrees well $\left(\chi^{2} / \nu \lesssim 1\right)$ with that observed at every redshift at which this comparison is possible, and the inverse (predicted merger MF from the QLF) also agrees well with the observations. Since we have shown that the buildup of the elliptical MF and $M_{50}$ trace a similar mass distribution to mergers, it is expected that the resulting $L_{*}$ will agree with the QLF. However, our comparison here, unlike in Hopkins et al. (2006e), is model independent, based only on the welldetermined BH-host mass relation and Eddington limit.

\subsection{The Transition Mass from the QLF Break}

In Figure 12 we invert this comparison and estimate the characteristic transition mass expected based on the observed QLF. We first show points as in Figure 11, estimating a characteristic host mass from the QLF $L_{*}$ (inverting eq. [6]). However, a proper calculation is not so trivial, as in detail $M_{50}$ will be determined by the integrated buildup of the early-type MF (and late-type MF for $M_{\mathrm{tr}}, M_{Q}$ ), and therefore requires that we adopt some model for quasar light curves and triggering. Hopkins et al. (2006a, 2006b) 


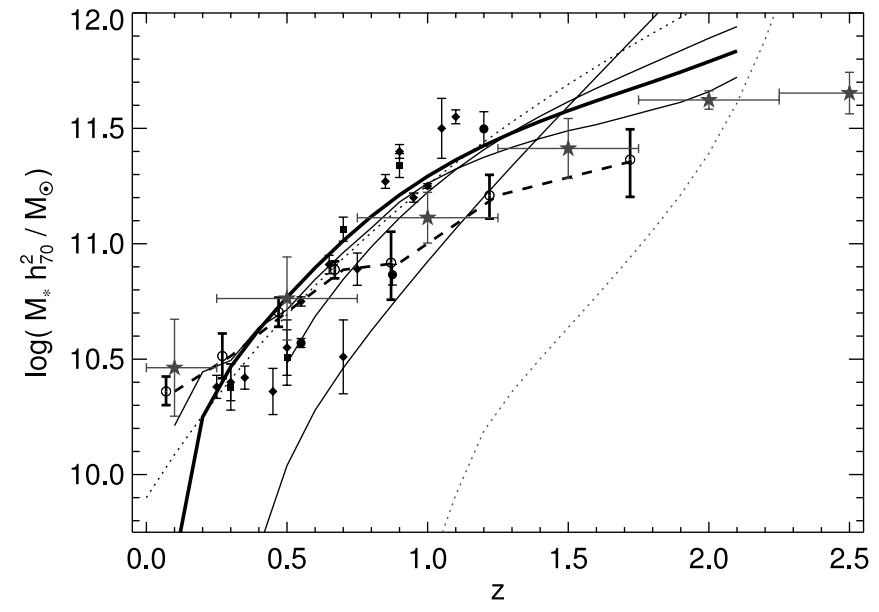

FIG. 12.-Observed transition mass (black points show $M_{50}$ from Fig. 2, from color-selected samples only, for clarity; dotted black line shows best-fit trend) compared with that predicted from QLFs. Stars show the observed QLF characteristic luminosity $L_{*}$ from Hopkins et al. (2007) (see Fig. 11; other samples not shown for clarity but trace a similar trend) directly converted to a characteristic associated mass given the observed Eddington ratios and $\mathrm{BH}$-host mass relation at these redshifts. Solid lines show the prediction of the simple assumption that the initial trigger of each bright quasar is associated with the formation/addition of an early-type galaxy. In detail, the compilation of QLF data from $z=0$ to 6 in Hopkins et al. (2007 and references therein) is converted therein to a massdependent rate of early-type formation/addition, given this assumption and the model Eddington ratio distributions (light curves) from Hopkins et al. (2005a, 2005b, 2005c, 2005d, 2006a, 2006b, 2006c, 2006d, 2006e). Thin lines use different model fits of this rate to the QLF data (and give an approximate idea of the uncertainties in this empirical modeling) to calculate $M_{50}$, with the thick line adopting the best fit to the quasar data (Hopkins et al. 2007; see their Table 5). Open circles and the dashed line calculate $M_{\text {tr }}$ (adopting the observed late-type galaxy MFs from Bell et al. [2003; $z=0.05$ ], Borch et al. [2006; $z=0.2-1.0$ ], and Fontana et al. [2004; $z=1.0-1.7])$ instead of $M_{50}$, with the same method. The gray dotted line calculates $M_{50}$ in this manner, but instead adopts an unphysical lightbulb quasar light-curve model (alternatively, this assumes that all observed low-luminosity quasars are in ongoing mergers/quenching). As in Fig. 11, the QLF break appears to trace the same parent population mass and evolution with redshift as $M_{\mathrm{tr}} / M_{50}$, consistent with quasar triggering and the buildup of $\mathrm{BH}$ mass being associated with the buildup of spheroid populations. [See the electronic edition of the Journal for a color version of this figure.]

use a large set of several hundred hydrodynamical simulations (Robertson et al. 2006a, 2006b) of galaxy mergers, varying the relevant physics, galaxy properties, orbits, and system masses, to quantify the quasar lifetime (and related statistics) as a function of the quasar luminosity. They define the quantity $t_{Q}\left(L \mid M_{\mathrm{BH}}\right)$, i.e., the time a quasar of a given $\mathrm{BH}$ mass [equivalently, peak quasar luminosity $\left.t_{Q}\left(L \mid L_{\text {peak }}\right)\right]$ will be observed at a given luminosity $L$. They further demonstrate that this quantity is robust across the wide range of varied physics and merger properties; for example, to the extent that the final $\mathrm{BH}$ mass is the same, any major merger of sufficient mass ratio (less than $\sim 5: 1$ ) will produce an identical effect. Given the tight $\mathrm{BH}$-host mass relation $\left(M_{\mathrm{BH}}-M_{*}\right)$, it is trivial to write this as $t_{Q}\left(L \mid M_{*}\right)$. Since at all $L, t_{Q} \ll t_{\mathrm{H}}$ (the Hubble time), the observed QLF $\phi_{Q}(L)$ is given by

$$
\phi_{Q}(L, z)=\int t_{Q}\left(L \mid M_{*}\right) \frac{d \Phi\left(M_{*}, z\right)}{d t d \log M_{*}} d \log M_{*},
$$

where $d \Phi\left(M_{*}\right) / d t d \log M_{*}\left[\right.$ hereafter $\left.\equiv \dot{\phi}\left(M_{*}, z\right)\right]$ is the rate of quasar triggering as a function of host spheroid mass at a given redshift. If the trigger is associated with formation of the spheroid or movement of a galaxy to the RS, then $\dot{\phi}\left(M_{*}, z\right)$ is the rate of buildup of the RS as a function of mass, directly comparable to that in Figure 4.
Knowing $\phi_{Q}(L)$ directly from observations and adopting the Hopkins et al. (2006a, 2006b) $t_{Q}\left(L \mid M_{*}\right)$ (which is at least consistent with all quasar observational constraints), the inversion of equation (7) yields $\dot{\phi}\left(M_{*}, z\right)$. Hopkins et al. (2007) perform this inversion, using their large compilation of observed $\phi_{Q}(L)$, and quote the best-fit $\dot{\phi}\left(M_{*}, z\right)$ (see their Table 5). We adopt their best-fit model for each redshift interval (individually, although a global fit yields similar results). If, again, this represents the buildup of the RS, then we can integrate from $z \rightarrow \infty$ to obtain the RS MF at all redshifts and calculate $M_{50}(z)$. The late-type MF is reasonably well measured over the range of interest, so comparing it with this integration also yields an expected $M_{\text {tr }}$ and $M_{Q}$. We compare these estimates with the observed $M_{50}$ in Figure 12 and find that they agree at all observed redshifts.

Having obtained the rate of buildup of early-type MFs expected if each quasar trigger is associated with the formation/ movement of an RS galaxy, we can directly compare with the rate of buildup implied by observed early-type MFs. Figure 13 plots the time-averaged buildup determined from the QLF, from the same $\dot{\phi}\left(M_{*}, z\right)$ as Figure 12, compared with the observed buildup from Figure 4. We consider both the mean time-averaged buildup (averaged over each appropriate redshift interval), assuming that each quasar trigger is instantaneously associated with the movement of a galaxy to the RS, and that expected if there is a uniform $\sim 1$ Gyr delay after each quasar before the galaxy becomes red (allowing time for, e.g., gas exhaustion and reddening). In either case, this estimate agrees with the observed buildup of elliptical populations, at all masses and redshifts $\left(\chi^{2} / \nu \sim 1\right.$ at all $z \gtrsim 0.3$ ). The latter ( $1 \mathrm{Gyr}$ delay) case gives marginally better agreement, but the difference between the two is comparable to the uncertainties in either determination of $\dot{\phi}\left(M_{*}, z\right)$ (see Fig. 12). At the highest masses at low redshifts $(z \lesssim 0.3$, although also to a lesser extent at $z \sim 0.3-0.7$ ), this estimate falls short of observed rates of buildup. However, this is precisely where we have estimated that observed rates of dry mergers can account for earlytype growth. Since gas-free mergers are not expected to trigger quasar activity, it is not surprising that this would not be implicit in QLFs. Allowing for the contribution of dry mergers shown at $z \lesssim 0.3$ improves the agreement considerably $\left(\chi^{2} / \nu \sim 2\right)$. There is still some tension matching the observations near $10^{11} M_{\odot}$, but it is important to note that at these redshifts, the cosmic variance associated with small-volume, narrow-field galaxy surveys and, perhaps more importantly, with even wide-field quasar surveys (given the very low local space density of quasars) is largest.

Having estimated the rate of quasar triggers, $\dot{\phi}\left(M_{*}, z\right)$, in Figure 13, then if each such trigger is in fact a galaxy merger, we can convert this to an expected merger fraction in exactly the same manner as we converted the rate of early-type buildup in Figure 4 (i.e., simply assuming an observable merger timescale $t_{\text {merger }}$ ). This is shown in Figure 9, along with the observed additional contribution from dry mergers. Given the agreement with the rate of elliptical buildup in Figure 13, it is not surprising to find that this agrees with observed merger fractions.

As a caution, we should note that these calculations can give a misleading result if the full luminosity dependence of the quasar lifetime from simulations (Hopkins et al. 2005a, 2005d, 2006b) and observations (e.g., Adelberger \& Steidel 2005b; Volonteri et al. 2006) is not properly taken into account. Such a case is not, of course, well motivated physically, although it may represent alternative quasar feedback models (or a complete lack of such feedback), but it is nevertheless sometimes adopted for simplicity. Why should such a simplified model give a qualitatively different result? In the $t_{Q}\left(L \mid M_{\mathrm{BH}}\right)$ model we consider, 


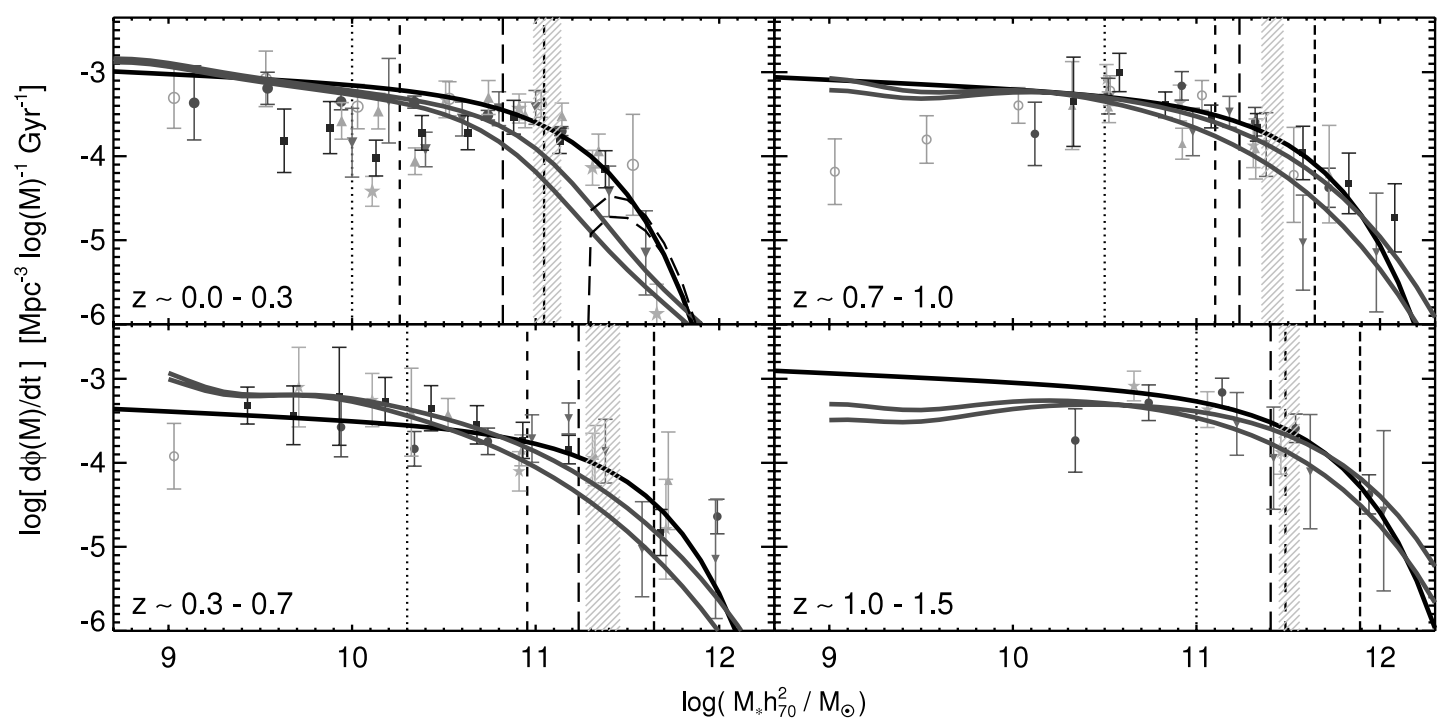

FIG. 13.-Time-averaged rate of buildup of early-type populations, as in Fig. 4, compared to that implied by the QLF if every quasar trigger is associated with the movement/formation/transition to the RS of a corresponding $\left(M_{*} \sim 10^{3} M_{\mathrm{BH}}\right)$ spheroid. The functional form for the QLF and implied triggering rate is taken from the best fit given in Hopkins et al. (2007). The lower line in each panel assumes instantaneous reddening, the upper line a 1 Gyr delay (postquasar peak) before objects appear on the RS. The implied rate, if quasars and the blue-red transition are associated with the same event, agrees well with the buildup of elliptical populations at all masses at moderate and high redshifts. At low redshift $z \lesssim 0.3$ (and to a lesser extent, at $z \sim 0.3-0.7$ ), the implied rate from the QLF falls below the observed buildup at high masses. The deficit can be accounted for at the highest masses with the observed rate of dry mergers (which, by definition, will not generally trigger quasar activity). [See the electronic edition of the Journal for a color version of this figure.]

$t_{Q}$ is larger at low luminosities because low-level AGN activity can persist for a long time after the violent, sudden high accretion rate episode in a merger. Ignoring this luminosity dependence and assuming, e.g., that all quasars turn on and off (as "lightbulbs") for a short time implies that all observed quasars, even those at very low luminosity, are seen at (or very near) their trigger, i.e., are in ongoing mergers. This gives a misleading estimate of the number of mergers needed to account for the QLF and, as a result, yields an incorrect estimate of host LFs and BH MFs (e.g.,
Hopkins et al. 2006b, 2006e), as well as, consequently, erroneous estimates of the associated transition mass.

\subsection{Further Tests of This Association}

Having considered the Hopkins et al. (2006b) models of merger-triggered quasar light curves, we briefly note additional future tests of these models and the generic association between the blue-red transition or elliptical formation and quasar activity. In Figure 14 we compare the observed QLF with the expected
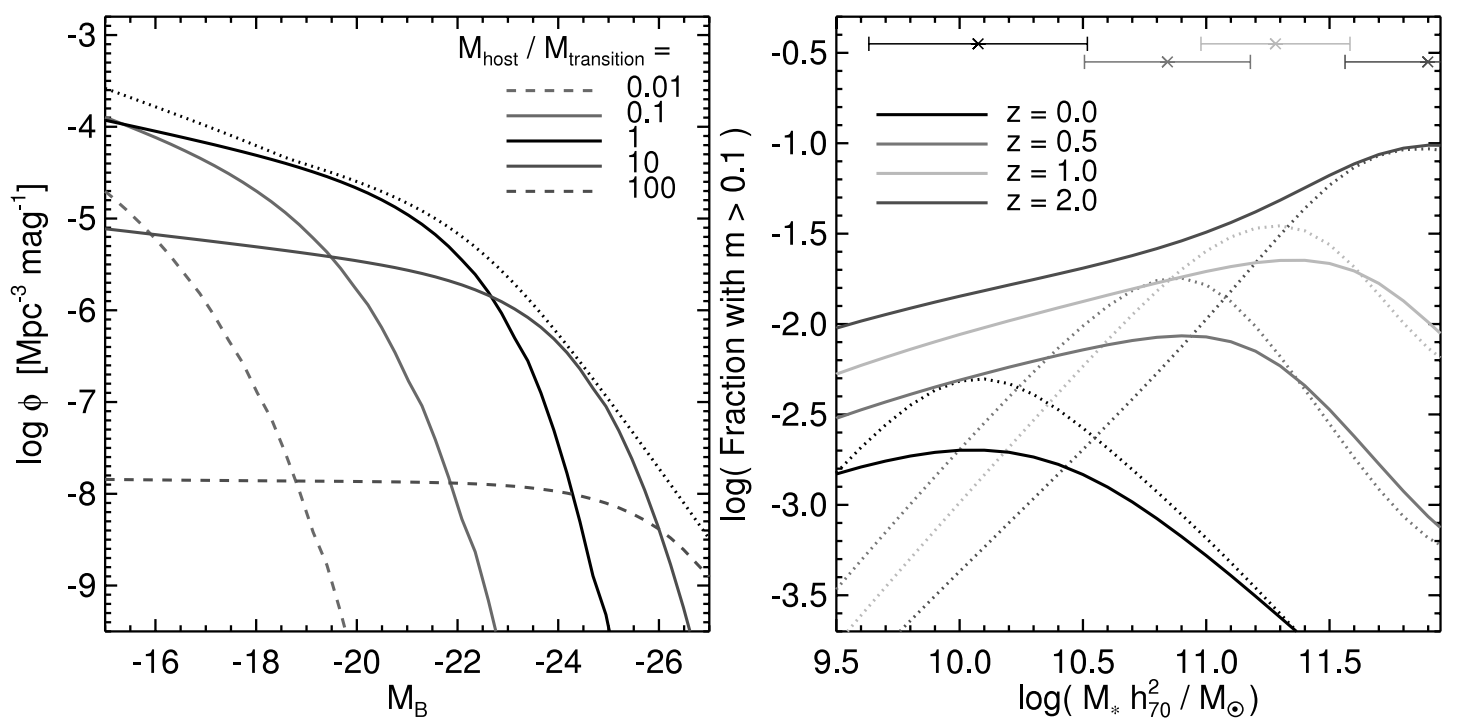

FIG. 14.-Left: Predicted contribution to the total B-band QLF (dotted line; from the compilation of Hopkins et al. 2007) from host galaxies in different mass intervals

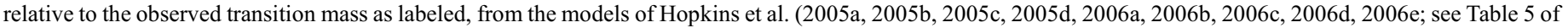

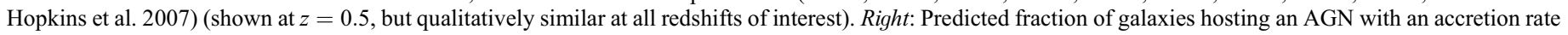

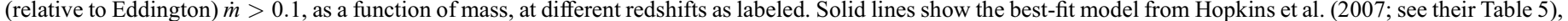

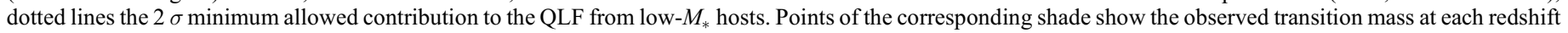

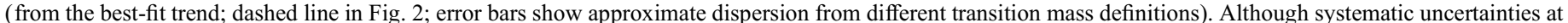

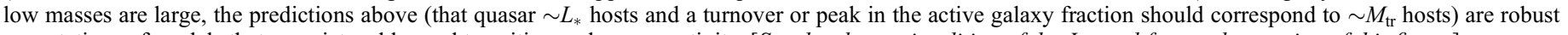
expectations of models that associate a blue-red transition and quasar activity. [See the electronic edition of the Journal for a color version of this figure.] 
conditional QLF, i.e., the contribution to the QLF from hosts/ merger remnants with different masses relative to the observed transition mass. In other words, the contribution

$$
\Delta \phi(L)=t_{Q}\left(L \mid M_{*}\right) \dot{\phi}\left(M_{*}, z\right) \Delta \log M_{*}
$$

from equation (7). The QLF near $L_{*}$ corresponds to objects with $M_{*} \sim M_{50}$. At the faintest and brightest luminosities, there are contributions from smaller and larger hosts, respectively (and a significant fraction of objects at the lowest luminosities will not necessarily be merger triggered; Hopkins \& Hernquist 2006), but it is clear from the figure that a direct measurement of the host masses of characteristic quasars at $z$ should find their hosts dominated by objects with $M_{\text {host }} \sim M_{\text {tr }}$ or $M_{50}$, many of which should appear as relatively young ellipticals, if this picture is correct.

In this scenario, one might also expect that objects around the transition mass preferentially host AGNs. Since equation (7) implicitly defines the probability to see a galaxy with mass $M_{*}$ (BH mass $M_{\mathrm{BH}} \sim \mu M_{*}$ ) at luminosity $L$, we can estimate the fraction of such galaxies with a given Eddington ratio. Figure 14 plots the fraction of galaxies hosting an AGN with an accretion rate (relative to Eddington) $\dot{m}>0.1$ as a function of galaxy stellar mass at several redshifts, compared to the observed transition mass at that $z$. [Note that the actual AGN luminosity will be $\dot{m} L_{\mathrm{Edd}}\left(M_{\mathrm{BH}}\right)$.] This particular prediction is sensitive to the different fits to the triggering rate $\dot{\phi}\left(M_{*}, z\right)$ provided in Hopkins et al. (2007) especially at low mass $\left(M \ll M_{50}\right)$, but the trend that the peak/turnover in this distribution tracks the transition mass is robust.

\section{THE TRANSITION MASS AND THE HALO QUENCHING MASS}

In most semianalytic models, gas infalling in dark matter halos is shock heated to the virial temperature and, in low-mass halos, subsequently cools on a short timescale, allowing rapid accretion onto the central halo galaxy and defining a "rapid cooling" or "cold accretion" regime. However, in massive halos, the cooling time is longer and gas forms a quasi-static hot halo, defining a "static hot halo" or "hot accretion" regime (e.g., Rees \& Ostriker 1977; Blumenthal et al. 1984). More recently, it has been suggested that the transition between these regimes is sharp, near a halo mass $M_{\text {crit }} \sim 10^{12} M_{\odot}$ (although this number is uncertain by a factor of several) at low redshift (Birnboim \& Dekel 2003; Kereš et al. 2005), and that suppression of future cooling and accretion is very efficient, essentially cutting off all gas supplies above this mass (e.g., Dekel \& Birnboim 2006) and quenching star formation.

The shock heating of infalling gas need not be the specific physical agent of this quenching: for example, radio-mode or low-luminosity, continuous AGN feedback (Croton et al. 2006; Cattaneo et al. 2006) or cyclic, short-lived quasar activity (Binney 2004) may be invoked to maintain the gas in the hot phase. There is therefore a potentially important distinction between semianalytic models (SAMs), which assume that the feedback mechanism is "at ready," such that on crossing the critical mass $M_{\text {crit }}$, star formation and gas accretion onto the central galaxy are instantaneously terminated, and those that require some additional mechanism or process (such as the formation of a relatively massive bulge and $\mathrm{BH}$ ) to drive the blue-red transition and transformation/ movement of galaxies to the RS.

This essentially relates to the important distinction, discussed in $\S 1$, between the mechanism by which galaxies become red/ elliptical and that by which they maintain their colors/low SFRs.
The key value of invoking this hot accretion regime in SAMs has been the ability to suppress star formation on timescales of order the Hubble time. However, although this could, in principle, be necessary to yield red galaxies at $z=0$, it does not automatically follow that it is sufficient. In other words, there may be other processes (e.g., mergers and/or quasars) that drive the blue-red transition and movement to the RS, and the hot accretion mode simply maintains these galaxies at their low SFRs.

One possible interpretation of the observed transition mass, perhaps the most naive, is that the transition mass simply represents the stellar mass hosted in $M_{\text {crit }}$ halos at each redshift. If we adopt the expected halo quenching mass $M_{\text {crit }}$ from Dekel \& Birnboim (2006) and assume either that the galaxies hosted have the same stellar mass as those in $z=0$ halos of the same mass (measured in Mandelbaum et al. 2006) or that they are already fully assembled (i.e., have stellar masses at $z$ appropriate for what their halo mass will be at $z=0$ ), we can compare with our observed transition mass. We find that while the two are similar at low redshifts, they diverge at higher $z$. This is, of course, where the observations are most uncertain, so it may simply reflect a systematic error in our estimation of the transition mass. But it probably also reflects the possibility that, in these models, the transition mass has a more complex physical origin than simply tracing $M_{\text {crit }}$. As noted in $\S 3$, allowing for more complex and realistic distributions of galaxies in transition to the RS can affect quantities such as the break mass $M_{*}$ and transition mass in a nontrivial manner. But there is also the possibility that the transition to the RS requires additional processes beyond the initial cutoff of new gas supplies in hot mode accretion, such as gas exhaustion, mergers, and/or quasar activity, to operate, which are what we see traced by the observed transition mass.

It is also worth considering whether or not the flow of galaxy host halos across $M_{\text {crit }}$ is consistent with the number and mass densities of transition objects and the buildup in early-type populations that we have estimated from the observations. The rate at which halos cross a given mass threshold $M$ is straightforward to calculate in linear theory from the Press-Schechter formalism,

$$
F(>M \mid z)=\operatorname{erfc}\left(\frac{\delta_{\text {coll }}(z)}{\sqrt{2} \sigma(M)}\right),
$$

and either adopting a simple mean stellar-to-dark matter mass ratio (from the calibration of Mandelbaum et al. 2006) or integrating (at this halo mass) over the population of inferred hosted galaxies from the conditional galaxy mass functions (CMFs; i.e., probability that halos of mass $M$ host galaxies of stellar mass $M_{*}$ ) yields an estimate of the rate at which stellar mass crosses this threshold. The local CMF is determined (albeit indirectly) entirely from observations of galaxy mass/luminosity functions and clustering (e.g., Yang et al. 2003, 2005; Zheng et al. 2005) and has been subsequently measured directly in 2dFGRS group catalogs by Yang et al. (2005) and is well constrained with typical uncertainties smaller than or comparable to those in our estimate of the rate of early-type buildup (at least for $M_{*} \gtrsim 10^{10} M_{\odot}$ of interest here; the MF at lower masses depends on the mass threshold for inclusion of satellite systems). Yan et al. (2003) and Cooray $(2005,2006)$ extend the conditional luminosity function (CLF)/ CMF to high redshifts $(z \lesssim 4)$ using a large number of LF and clustering estimates from wide-area surveys. Note that Yang et al. (2003, 2005) and Cooray (2006) actually measure the CLF; we convert to a CMF using the appropriate $M / L$ ratios as a function of mass from Bell et al. (2003) and assume that these $M / L$ values evolve with redshift following the best-fit stellar population 

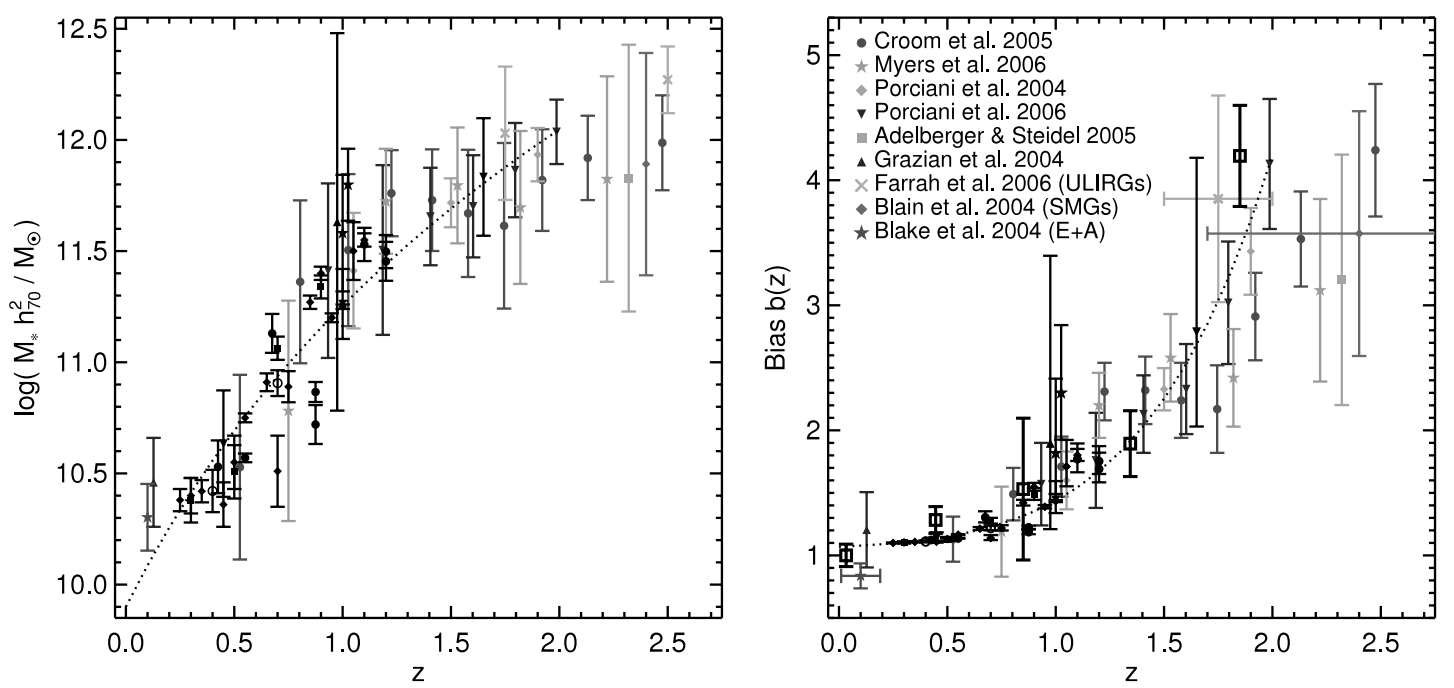

FIG. 15.-Left: Observed transition mass (black points show $M_{50}$ as in Fig. 2, from all samples; black dotted line shows best-fit trend) as a function of redshift, compared with the characteristic host masses (shaded points) of quasars, recently formed elliptical (E+A/K+A) galaxies, and ongoing bright mergers (ULIRGs/SMGs), estimated from their clustering properties. Right: Corresponding bias as a function of redshift. Black points as in left panel show $b(z)$ calculated from $M_{50}(z)$ and the observed $z=0$ bias ( $\mathrm{Li}$ et al. 2006) for that stellar mass (i.e., assuming passive evolution); open black squares show $b(z)$ determined directly from observations for red galaxies with the appropriate $M_{50}(z)\left[M_{50}(z)\right.$ from best-fit trend; points of increasing redshift from Li et al. 2006; Shepherd et al. 2001; Pollo et al. 2006; Meneux et al. 2006; Brown et al. 2005, respectively]. Shaded points show $b(z)$ observed for the quasar, E+A, and merger populations, as labeled. Note that $b(z)$ is defined from the clustering length $r_{0}$ and thus the relative bias and $M_{*}$ shown are only weakly cosmology dependent. This provides a completely independent check of the previous comparison between these populations, but one that suggests a similar coevolution. [See the electronic edition of the Journal for a color version of this figure.]

models as a function of mass from Gallazzi et al. (2006) and Renzini (2006). Checking directly (following the methodology of Yang et al. 2003) shows that this agrees with the Bell et al. (2003) MFs (see also their Fig. 19) and the Li et al. (2006) measured clustering as a function of stellar mass; furthermore, these CMFs agree well with those directly determined in Zheng et al. (2005). Ultimately, there are a number of systematic (factor of $\sim 2-3$ ) uncertainties in this comparison, and our (admittedly crude) empirical calculation ignores the fact that, in quenching models, crossing the quenching threshold itself may change the stellar-to-dark matter mass ratios and stellar $M / L$ values. However, within these rather large uncertainties, our purely empirically estimated rate at which galactic host halos cross $M_{\text {crit }}$ is consistent with the possibility that this is a necessary prerequisite for transition to the RS.

\section{CLUSTERING: AN INDEPENDENT TEST}

We compare the populations we have considered in an independent manner by examining their clustering properties. If a population (i.e., a given set of parent halos) clusters with a given bias $b(z)$ at some redshift $z$, then the subsequent evolution in their bias is trivially calculated in linear theory

$$
b(z=0)=1+D(z)[b(z)-1],
$$

where $D(z)$ is the growth factor (Croom et al. 2001), regardless of the processes (accretion, mergers, etc.) that affect the halos (and galaxies) themselves. The bias of galaxies (specifically red/ elliptical galaxies) as a function of stellar mass is well determined at $z=0$ (we adopt the recent determination from the SDSS in Li et al. [2006], with typical $\lesssim 10 \%$ uncertainty), so given the bias of a population at $z$ and evolving it to $z=0$ with equation (10) yields the characteristic $z=0$ stellar mass of this population (i.e., the average stellar mass of which the population is the parent).

Figure 15 shows this $M_{*}$, calculated from various clustering measurements $b(z)$ of quasars, mergers (ULIRGs and submilli- meter galaxies [SMGs]), and E+A galaxies as a function of redshift, and compares to the transition mass $M_{50}$ at each redshift. We invert this as well; knowing $M_{50}(z)$, evolve $b\left(M_{50}, z=0\right)$ with equation (10) to estimate $b(z)$. Note that all $b(z)$ shown from measurements are converted from the directly observed clustering length $r_{0}$, which for a power-law correlation function yields

$$
r_{0}=r_{0}(z=0)[b D(z)]^{\gamma / 2},
$$

with $r_{0}(z=0) \sim 5 h^{-1} \mathrm{Mpc}$ and $\gamma \sim 1.8$ (see, e.g., Norberg et al. 2002). The absolute value of the bias as plotted is then weakly dependent on cosmology (and this conversion, of course, is inexact), but the important point for our purposes is that the relative bias of all points plotted [and $b\left(M_{*}, z=0\right)$ with which we compare] is insensitive to the cosmology.

These comparisons do assume that the stellar mass of individual systems does not change much from $z$ to $z=0$, i.e., that, once formed, ellipticals are passively evolving. However, we can easily eliminate this assumption, by considering the clustering directly observed for red galaxies of mass $M_{50}(z)$ at that redshift, and Figure 15 shows this as well. In either case, the agreement with the clustering of quasars and (albeit much less well constrained) merger/E+A populations is good. This also agrees with determinations of, e.g., the typical overdensities and smallscale clustering of quasars and ULIRGs (Farrah et al. 2004; Hennawi et al. 2006; Serber et al. 2006).

This method by which we compare clustering is only weakly dependent on cosmology, through the growth factor $D(z)$ (independent of, e.g., $\sigma_{8}$ ). There are some caveats, however. Technically, we are estimating the mass that has exactly the observed bias; this is some weighted mean mass. However, theoretical expectations from physically motivated quasar light-curve models (Lidz et al. 2006) and direct observations of clustering as a function of luminosity (Adelberger \& Steidel 2005b; Croom et al. 2005; Myers et al. 2006) suggest that quasar clustering depends only weakly on luminosity, reflecting a reasonably well defined characteristic host mass. These comparisons will also, of course, 
be affected if the clustering of mergers is different on large scales from that of nonmerging halos of the same mass (a so-called merger bias). However, a number of investigations have found no such dependence (e.g., Lemson \& Kauffmann 1999; Kauffmann \& Haehnelt 2002; Percival et al. 2003), and even where more recent investigations have seen such an effect (Gao et al. 2005) it has been restricted to small-mass halos (below the collapse mass, i.e., where $b=1$ ) at $z=0$, and therefore the assumption of no merger bias has generally been adopted in quasar clustering studies (Martini \& Weinberg 2001; Haiman \& Hui 2001; Adelberger \& Steidel 2005b; Croom et al. 2005; Myers et al. 2006; Lidz et al. 2006).

We can repeat this comparison using the formalism of Mo \& White (1996) from linear collapse theory, which yields a characteristic halo mass from a given observed $b(z)$. We use the observed stellar mass-halo mass relations calibrated for elliptical galaxies from weak-lensing measurements in Mandelbaum et al. (2006) to convert these halo masses to a stellar mass $M_{\mathrm{tr}}$. We convert between halo mass and bias with the method of Mo \& White (1996) modified following Sheth et al. (2001) in our adopted cosmology (in detail assuming $\sigma_{8}=0.8, n_{s}=0.98$ ) with the power spectrum computed following Eisenstein \& $\mathrm{Hu}$ (1999). The results are similar but are much more sensitive to the adopted cosmology and systematics in the stellar mass-halo mass relation in this approach.

In considering the clustering of $M_{50}$ objects, we have considered the directly measured bias of objects with mass $M_{50}(z)$ at redshift $z$, as well as the passively evolved clustering from the $z=0$ bias as a function of mass. We can gain further insight into the evolution of these populations by comparing the two. Knowing the observed bias of $M_{50}(z)$ objects at $z$, we can evolve this to $z=0$ given equation (10) and then use $b\left(M_{*}, z=0\right)$ to obtain the typical stellar mass hosted by these systems at $z=0$. Comparing that to their stellar mass at $z$, namely, $M_{50}(z)$, shows by how much the typical stellar mass of the population has grown. We could also estimate this in a more indirect fashion, using linear theory to estimate a host halo mass $M_{\text {halo }}(z)$ given $b(z)$, and then, knowing the $z=0$ mass of a halo with mass $M_{\text {halo }}(z)$ at $z$, use the local galaxy stellar-halo mass calibrations from Mandelbaum et al. (2006) to obtain $M_{*}(z=0)$. Again, this approach is considerably more sensitive to the assumed cosmology, but in our adopted case it yields similar results.

In Figure 16 we use this to compare $M_{50}(z)$, the stellar mass of transition mass objects at $z$, with $M_{*}(z=0)$, i.e., the mean $z=0$ stellar mass that is typically hosted by the evolved parent halos. Unless transition mass objects comprise some unusual outlier in their halo properties, this should represent the typical stellar mass these objects will grow to by $z=0$. We compare with the expectation, following, e.g., Bell et al. (2006b), that these stellar masses grow at a rate corresponding to one major (mass ratio $1: 1$ ) merger since $z=1$. We also consider the case if the stellar mass in these objects grows in fixed proportion with their host dark matter halos.

The systematic uncertainties [and measurement errors in $b(z)$ ] are sufficiently large that we should regard these comparisons with caution and not consider this as evidence for a particular amount of dry merging. However, the estimated $M_{*}(z=0)$ demonstrates that the $M_{50}(z)$ measurements are completely consistent with subsequent growth by dry mergers at observationally inferred rates. Growth in proportion to the host halo mass, by contrast, is extremely difficult to reconcile with observed properties of the galaxies. This is not surprising, as the existence of any significant $\sim 10^{12} M_{\odot}$ galaxy population at $z=1$ without a corresponding $\sim 10^{13}-10^{14} M_{\odot}$ galaxy population at $z=0$ implies that, at least

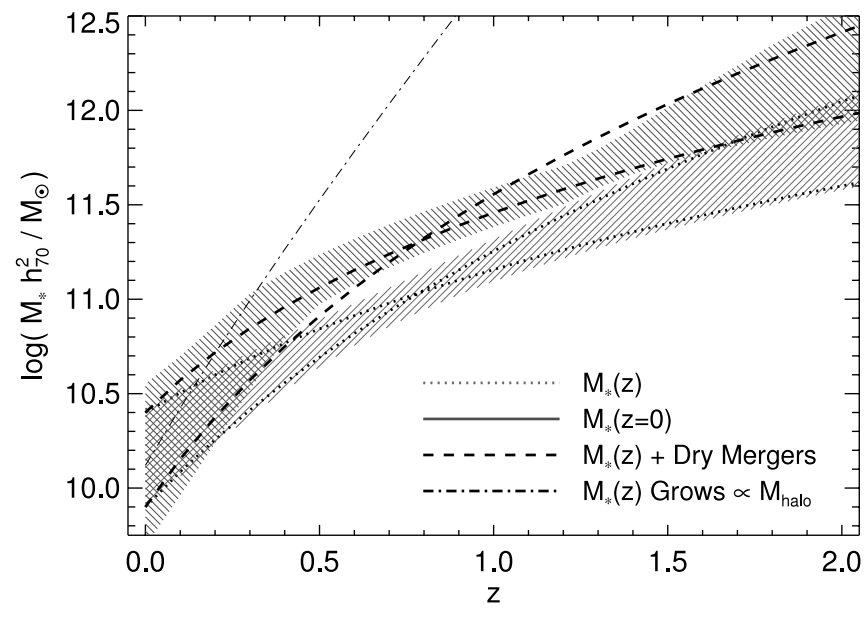

FIG. 16.-Best-fit transition stellar mass $M_{50}(z)$ (see Fig. 2; dotted lines with shaded range show $\sim 1 \sigma$ range) at a given redshift, compared to the inferred stellar mass of the same objects at redshift $z=0$ (upper shaded range). The observed bias of a fixed set of objects (halos) with stellar mass $M_{50}(z), b\left(M_{50}(z), z\right)$, evolves simply to $z=0$ (Croom et al. 2001), where a comparison with the local $b\left(M_{*}, z=0\right)$ yields the typical stellar mass hosted by such halos. Dashed lines show the result of taking $M_{50}(z)$ (dotted lines) and allowing for a constant rate ( $\sim 1$ since $z=1$, as suggested by observations; van Dokkum 2005; Bell et al. $2006 \mathrm{~b}$ ) of major (equal mass) dry mergers. The dot-dashed line assumes that subsequent (after $z$ ) galaxy assembly tracks halo assembly (i.e., efficient dry merging; no downsizing in assembly times), as implied by some semianalytic models (e.g., De Lucia \& Blaizot 2007; Cattaneo et al. 2006; note that this is not a rigorous comparison with these models). Although systematic uncertainties are sufficiently large that this should not be considered evidence for a particular amount of dry merging, the comparison demonstrates that the evolution in $M_{50}(z)$ is completely consistent with observational evidence for a significant growth by dry mergers since $z \gtrsim 1$. A very large number of dry mergers is, however, inconsistent, and this also violates the direct observationally determined rates of dry mergers above (galaxies grow by a factor $\gtrsim 2$ since $z=1$ ). Note that dry mergers do not, by definition, build up the total mass budget on the RS and, at observed rates, have a relatively weak effect on the RS MF near the transition mass (see also Fig. 4). [See the electronic edition of the Journal for a color version of this figure.]

for some objects assembled most rapidly, subsequent galaxy assembly must lag behind halo growth (or subsequent growth in these halos must be anomalously slow). This does mean, however, that it is not possible to reconcile the observations with a model in which galaxy assembly uniformly tracks halo assembly, even allowing for the final galaxy stellar-halo mass ratio to be a function of halo mass (i.e., setting in all progenitors the effective $M / L$ of the $z=0$ halo, which then simply assembles).

\section{SUMMARY AND DISCUSSION}

We compile a large number of observations of red/elliptical galaxy MFs and use these to determine the rate of buildup of the $\mathrm{RS}$ as a function of mass and redshift. Comparing these with observations of other populations allows us to test a number of different models for the possible associations between these populations and the transition of galaxies from blue, star-forming disks to red, dead ellipticals.

Independent of the nature of downsizing in the buildup of RS MFs (discussed below), the rate of RS buildup is sufficiently well determined to place meaningful constraints on a number of models. Dissipationless (gas-poor, red, or dry) mergers can account for the buildup of the RS at only the largest masses $\gtrsim 10^{11} M_{\odot}$ at low redshift $(z \lesssim 0.3)$. At higher redshifts $(z \gtrsim 0.5)$, the dry merger rate would have to be at least an order of magnitude larger than observationally estimated (van Dokkum 2005; Masjedi et al. 2006; Bell et al. 2006a, 2006b; Lotz et al. 2006b) to account for observed RS buildup, even at the highest masses. This is perhaps unsurprising, as these and other observations 


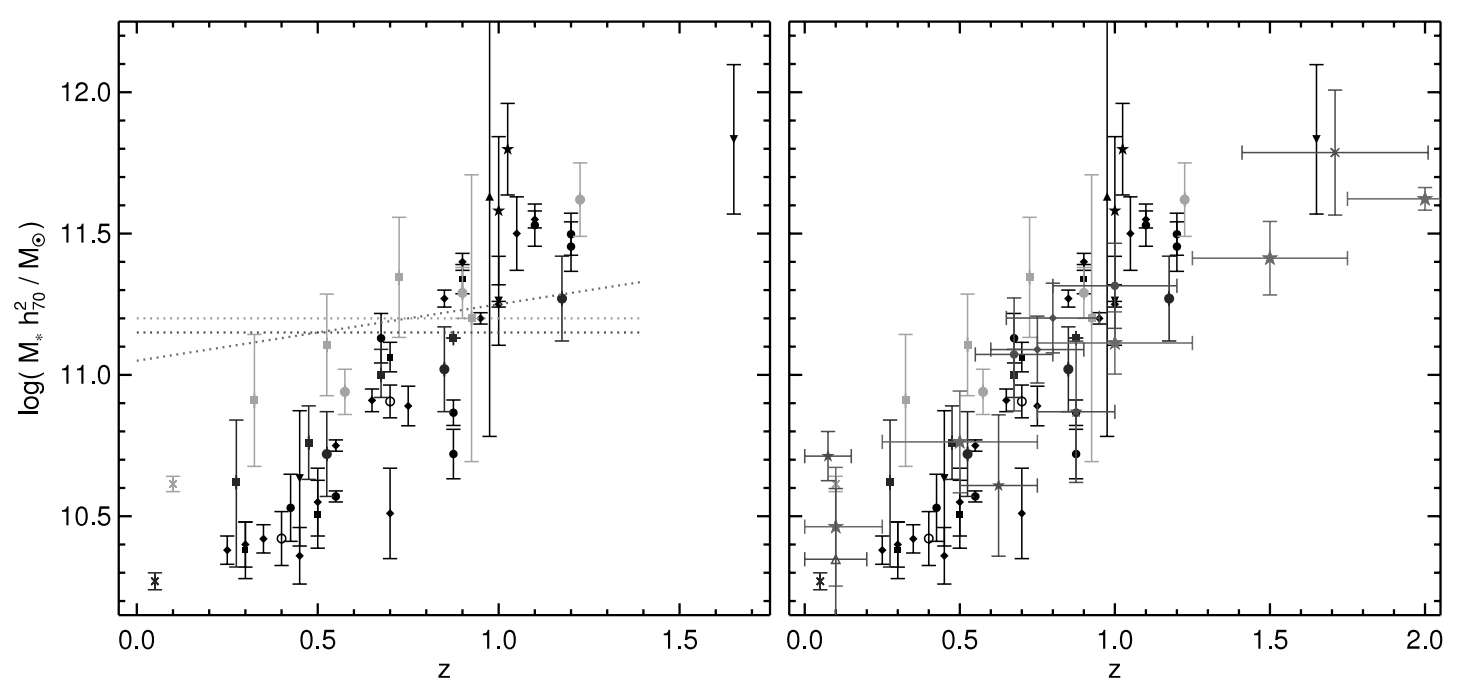

FIG. 17. - Left: Transition mass determined by various definitions ( $M_{50}$ from all samples, $M_{\text {tr }}$ from color-selected samples, and $M_{Q}$ from color-selected samples, as in Fig. 2), as a function of redshift. Dotted lines show the characteristic mass $M_{*}$ of all, red, and blue galaxies (see Fig. 5). The null hypothesis that transition mass objects (as well as quasars and mergers; see right panel) are drawn randomly/uniformly from the all, red, or blue galaxy population can be ruled out at $>6 \sigma(>5 \sigma$ for quasars, $>3 \sigma$ for mergers). Right: Transition mass, as in the left panel, together with the characteristic masses $M_{*}$ of merger MFs ( points; as in right panel of Fig. 7) and quasar hosts (stars; as in Fig. 12). The observations at all redshift are consistent with the hypothesis that mergers, quasars, and the transition/addition to the RS are associated with the same event. [See the electronic edition of the Journal for a color version of this figure.]

find that the gas-rich merger rate/fraction is an order of magnitude or more larger at all but the lowest redshifts. Furthermore, the total mass density on the RS is observed to increase by a factor of $\sim 2.5-3$ since $z \sim 1$ (e.g., Bundy et al. 2005; Franceschini et al. 2006; Pannella et al. 2006; Borch et al. 2006), and dry mergers cannot, by definition, move/form new galaxies and mass on the RS.

However, we find that the total observed merger population (gas-rich+gas-poor) agrees very well with that expected if all RS galaxies are formed in mergers. Both the detailed mass distribution and fraction/rates of galaxy mergers are consistent with the rate of RS buildup at all masses and redshifts observed. This merger population is dominated by gas-rich mergers at all masses at high redshifts $(z \gtrsim 0.5$; Bell et al. 2006a; Lotz et al. 2006b) and at low masses at low redshifts, morphologically identifiable as bright (i.e., star-forming or starbursting) interacting systems (e.g., Bundy et al. 2006; Wolf et al. 2005). In detail, completely neglecting dry mergers (or merger MFs sensitive to them), this agreement is unchanged except for the highest masses at low $z$ discussed above. There is substantial systematic uncertainty in converting a merger fraction to a merger rate; our comparisons assume a characteristic observable merger timescale of $\sim 0.5$ Gyr. However, this is a theoretically reasonable timescale (see $\S 4$ ), and given the scatter in the observations, our conclusions are not changed for systematic shifts within a factor of $\lesssim 2$, nor for allowing the merger timescale to scale with halo dynamical times $\left[\propto(1+z)^{-3 / 2}\right]$. Furthermore, this has no effect on our comparison of the mass distributions of these populations.

Similarly, we find that the rate at which host galaxies trigger quasars, determined as a function of the host stellar mass and redshift from the QLF, agrees well with the observed RS buildup at all masses and redshifts observed. There is some discrepancy at the lowest redshifts and highest masses, but this is again where the dry merger contribution can account for the observed buildup, and dry mergers (by definition being gas-poor or gas-free) are not expected to trigger quasar activity. We consider this comparison first in a purely empirical fashion, using observed quasar Eddington ratios and the $\mathrm{BH}$-host mass relation to estimate quasar host masses as a function of redshift, and then in greater detail adopting the models of quasar light curves and lifetimes as a function of luminosity and host properties from the simulations of merger-induced quasar activity in Hopkins et al. (2006b). The latter introduces some model dependence (although it is consistent with the Eddington ratio and $\mathrm{BH}$-host mass relation estimates) but allows us to consider this comparison in greater detail and to make specific predictions for the characteristic host masses of quasars as a function of their position on the QLF and for the AGN or active fraction of galaxies as a function of stellar mass. In either case, the agreement between the rates of quasar formation/ triggering as a function of host stellar mass and the buildup of RS galaxies is similar.

We independently test these possible associations by comparing clustering measurements of the relevant populations as a function of redshift and find similar results. The clustering of quasars and systems in transition to the RS agree at all redshifts as if they trace the same mass distribution. Clustering properties of merger (ULIRG and SMG) and postmerger (E+A) populations are consistent, but considerably less well constrained.

Although the above comparisons do not technically depend on it, we determine the transition mass $\left(M_{\mathrm{tr}}, M_{Q}\right)$, i.e., the mass that separates the blue, star-forming disk and red, non-star-forming elliptical populations, as a function of redshift. It has been suggested (e.g., Bundy et al. 2006) that this represents the characteristic mass at which galaxies are forming on or being added to the RS as a function of redshift, but quantified in this manner, it is not obviously so (see, e.g., Shankar et al. 2006). We therefore also determine $\left(M_{50}\right.$, following Cimatti et al. 2006) the minimum mass above which the RS MF is $\gtrsim 50 \%$ assembled at a given redshift. Regardless of definition, and furthermore regardless of the criterion used to separate early- and late-type populations (whether, e.g., a color, SFR, or morphology criterion), $M_{\mathrm{tr}} / M_{Q} / M_{50}$ shift to systematically larger masses at higher redshift (significant at $>6 \sigma$ ), tracing a very similar trend as a function of redshift.

This trend, especially in $M_{50}$ (which is independent of possible evolution in late-type MFs), suggests that downsizing applies not just to galaxy star formation, but also in some sense to galaxy assembly, as suggested by the studies of, e.g., Bundy et al. (2005, 2006), Zucca et al. (2006), Yamada et al. (2005), Franceschini 
et al. (2006), Cimatti et al. (2006), Fontana et al. (2006), and Brown et al. (2007). In greater detail, considering the full rate of RS buildup as a function of stellar mass and redshift, lowmass ( $\lesssim 10^{11} M_{\odot}$ ) galaxies appear to be building up rapidly/ continuously at low redshifts $\left(\sim 7 \%-15 \% \mathrm{Gyr}^{-1}\right)$, but the most massive systems do not $\left(\sim 1 \% \mathrm{Gyr}^{-1}\right.$ growth at $\left.z \sim 0\right)$. The growth of the most massive systems instead appears to be rapid at significantly higher redshifts (e.g., rising to $\sim 20 \%-50 \% \mathrm{Gyr}^{-1}$ by $z \sim 1$ ). Equivalently, the characteristic mass (Schechter function $M_{*}$ ) defined by this formation rate appears (albeit at only $\sim 2-3 \sigma$ ) to increase with redshift in a similar fashion to the transition mass.

We compare the transition mass with the characteristic masses of mergers and quasars and again find that they trace similar masses as a function of redshift, with downsizing evident in all three populations ( $>3 \sigma$ for mergers, $>6 \sigma$ for quasars), further supported by their observed clustering. We compare with the characteristic (Schechter function) $M_{*}$ of the entire, red, and blue galaxy populations and rule out at high significance the possibility that transition mass objects are drawn uniformly from any of these populations. Even with the systematic uncertainties in this mass estimate, it is also clearly distinct as a function of redshift from the characteristic masses of, e.g., cluster, radio galaxy, ERO, DRG, or LBG populations (see, e.g., Fig. 2 of Farrah et al. 2006).

These observations are all consistent with and suggest a scenario in which major mergers, quasars, and the transition from blue disk to red elliptical galaxies are associated. They do not inform us regarding, for example, whether gas exhaustion or stellar or quasar feedback is the specific mechanism for the reddening that accompanies the merger-driven morphological transformation and quasar episode. However, they support the hypothesis that mergers drive the transition from blue disks to red elliptical galaxies, terminating in decaying, feedback-driven bright quasar phases. The transition mass and break in the QLF appear to reflect the characteristic mass of gas-rich objects merging at a given redshift, which may build up the new mass on the RS at progressively lower masses at lower $z$ as gas supplies are exhausted in more massive systems.

That quasar host masses trace the transition mass and not, e.g., the blue galaxy population $M_{*}$ (see also Fig. 17) rules out the possibility that quasar activity generically traces star formation, as variants of, e.g., the Granato et al. (2004) models might predict. Likewise, it rules out the possibility that quasar masses generically trace the red galaxy $M_{*}$, as they would if quasar activity was long lived or randomly (but uniformly as a function of mass) episodic in high-mass BHs. The former case would be expected from the low-level AGN activity invoked in, e.g., Croton et al. (2006) if radio and optical or high Eddington ratio quasar activity were associated, but they are in fact generally believed to be distinct (e.g., Ho 2002; White et al. 2007; Körding et al. 2006). The latter case implies strong limits on implementations of, e.g., the Binney (2004) model, which seek to suppress cooling flows through sporadic but potentially high accretion rate AGN activity.

Although we can rule out some alternatives to the merger scenario, there remain a number of viable variants of quenching models, in which crossing the critical halo mass $M_{\text {crit }}$ and entering a hot accretion regime plays a key role in the transition to the RS. Especially given that some feedback mechanism is typically required, even in the hot accretion regime, to prevent the formation of cooling flows, it is easy to imagine a scenario in which, on entering this regime, new infalling halo gas is shock heated, but feedback from, e.g., a central disk galaxy with a small $\mathrm{BH}$ is inefficient, cold gas reservoirs remain large, and cooling flows can form. Thus, the system will not redden until it subsequently undergoes a major merger, which morphologically transforms the system, rapidly exhausts the remaining cold gas reservoir, and triggers a quasar and builds up a massive $\mathrm{BH}$, injecting some level of feedback and enabling efficient future (e.g., cyclic AGN or radio-mode) feedback. The hot accretion regime may be a necessary prerequisite for feedback to efficiently prevent subsequent cooling, and as discussed in $\S 7$, our comparisons are all consistent with this possibility. This is generally similar to the scenario assumed in, e.g., Croton et al. (2006) although they do not explicitly incorporate quasar light curves or feedback. Recognizing these distinctions (as opposed to, e.g., assuming that a system simply "shuts down" on reaching $M_{\text {crit }}$ ) will probably have little effect on the $z=0$ predictions of semianalytic models, since in either case star formation will be effectively suppressed at relatively early times in the most massive systems (see also Cattaneo et al. 2006). However, at higher redshifts when massive objects are still forming, the distinctions will almost certainly be significant.

We note that none of our conclusions conflict with the hypothesis that, once formed, elliptical galaxies can continue to grow by dry mergers. However, they emphasize that the importance of such mergers is restricted to the most massive galaxies at low redshifts. Our results, even the steep evolution of $M_{50}$ implying some downsizing in red galaxy assembly, are all consistent with (and, in fact, marginally favor) the relatively low observationally inferred dry merger rate $(\sim 1$ major dry merger since $z \sim 1$ ). Essentially, downsizing in galaxy assembly as we have quantified it is not, strictly speaking, "antihierarchical." Massive galaxies still continue to build up their populations to the present; it is simply a statement that the relative rates of red galaxy formation/assembly decrease or "slow down" at late times in the most massive systems. This could be related to pure dark matter processes, for example, the rapid evolution in large overdensities could simply exhaust the supply of galaxies with which to merge, or the cluster environments of massive systems at low redshift attain sufficient circular velocities as to rapidly reduce merger rates (see also Neistein et al. 2006). The evolution of the transition mass may, alternatively, be a statement that galaxy assembly does not strictly trace halo assembly. There are a number of baryonic processes that make this possible, as it simply requires that the effective baryon conversion efficiencies in galaxies be a function of time, or different for central versus satellite systems.

Improved measurements of early-type MFs at high redshift $(z \sim 1)$, larger samples of mergers from which to construct merger MFs, revised or direct determinations of high-redshift conditional MFs, and direct observations of the masses of quasar hosts will substantially improve the constraints in this paper. Ultimately, the integration of the merger and quasar host MFs may enable a purely observational comparison with the remnant, red galaxy MF. Calibration of the observable merger timescale with realistic high-resolution galaxy merger simulations, i.e., calibration of selection efficiencies for observed merger fractions, can further remove the factor $\sim 2$ uncertainty in comparing the rates of elliptical buildup and observed merger populations. The association favored here between mergers, quasars, and elliptical buildup also makes specific predictions for the characteristic masses of $\mathrm{E}+\mathrm{A}$ galaxies and quasar hosts as a function of redshift, which should be testable in future wide-field surveys.

The scenario we have described does not, of course, imply that mergers, quasars, and remnant ellipticals will necessarily be recognizable as the same, singular objects at a given instant; in fact, simulations that follow the transition through these stages (e.g., 
Hopkins et al. 2006c) predict that they will be seen as distinct phases in merger-triggered evolution, and observations tracking, e.g., the associations between dynamical merger state and quasar activity (e.g., Straughn et al. 2006) support this distinction. What we ultimately find evidence for here in the masses, luminosities, and clustering properties of mergers, galaxies being added or in transition to the RS, and quasars is that they are drawn from the same parent population, and that this population is distinct from the quiescent all/red/blue galaxy population. Again, none of this strictly implies causality, but it does favor models that associate these populations with the same event, a natural expectation if mergers of gas-rich galaxies trigger quasars and morphologically transform disks to spheroids, moving new mass to the early-type population and leaving an elliptical, gas-poor, rapidly reddening remnant galaxy.

We thank Eric Bell, Arjun Dey, David Hogg, Casey Papovich, Rachel Somerville, Stijn Wuyts, Sandy Faber, and Marijn Franx for very helpful discussions. We also thank T. J. Cox, Brant Robertson, and the anonymous referee, whose comments improved this manuscript. This work was supported in part by NSF grants ACI 96-19019, AST 00-71019, AST 02-06299, and AST 03-07690 and NASA ATP grants NAG5-12140, NAG5-13292, and NAG5-13381.
Adelberger, K. L., \& Steidel, C. C. 2005a, ApJ, 627, L1 2005b, ApJ, 630, 50

Alexander, D. M., Bauer, F. E., Chapman, S. C., Smail, I., Blain, A. W., Brandt, W. N., \& Ivison, R. J. 2005a, ApJ, 632, 736

Alexander, D. M., Smail, I., Bauer, F. E., Chapman, S. C., Blain, A. W., Brandt, W. N., \& Ivison, R. J. 2005b, Nature, 434, 738

Balogh, M. L., Baldry, I. K., Nichol, R., Miller, C., Bower, R., \& Glazebrook, K. 2004, ApJ, 615, L101

Barnes, J. E., \& Hernquist, L. 1991, ApJ, 370, L65 1992, ARA\&A, 30, 705 1996, ApJ, 471, 115

Baugh, C. M., Lacey, C. G., Frenk, C. S., Granato, G. L., Silva, L., Bressan, A., Benson, A. J., \& Cole, S. 2005, MNRAS, 356, 1191

Bell, E. F., McIntosh, D. H., Katz, N., \& Weinberg, M. D. 2003, ApJS, 149, 289

Bell, E. F., Phleps, S., Somerville, R. S., Wolf, C., Borch, A., \& Meisenheimer, K. 2006a, ApJ, 652, 270

Bell, E. F., et al. 2004, ApJ, 608, 752 2006b, ApJ, 640, 241

Benson, A. J., Bower, R. G., Frenk, C. S., Lacey, C. G., Baugh, C. M., \& Cole, S. 2003, ApJ, 599, 38

Berrier, J. C., Bullock, J. S., Barton, E. J., Guenther, H. D., Zentner, A. R., \& Wechsler, R. H. 2006, ApJ, 652, 56

Binney, J. 2004, MNRAS, 347, 1093

Birnboim, Y., \& Dekel, A. 2003, MNRAS, 345, 349

Blake, C., et al. 2004, MNRAS, 355, 713

Blumenthal, G. R., Faber, S. M., Primack, J. R., \& Rees, M. J. 1984, Nature, 311, 517

Borch, A., et al. 2006, A\&A, 453, 869

Borys, C., Smail, I., Chapman, S. C., Blain, A. W., Alexander, D. M., \& Ivison, R. J. 2005, ApJ, 635, 853

Bower, R. G., Benson, A. J., Malbon, R., Helly, J. C., Frenk, C. S., Baugh, C. M., Cole, S., \& Lacey, C. G. 2006, MNRAS, 370, 645

Boyle, B. J., Shanks, T., Croom, S. M., Smith, R. J., Miller, L., Loaring, N., \& Heymans, C. 2000, MNRAS, 317, 1014

Brinchmann, J., et al. 1998, ApJ, 499, 112

Brown, M. J. I., Dey, A., Jannuzi, B. T., Brand, K., Benson, A. J., Brodwin, M., Croton, D. J., \& Eisenhardt, P. R. 2007, ApJ, 654, 858

Brown, M. J. I., Jannuzi, B. T., Dey, A., \& Tiede, G. P. 2005, ApJ, 621, 41

Bruzual, G., \& Charlot, S. 2003, MNRAS, 344, 1000

Bundy, K., Ellis, R. S., \& Conselice, C. J. 2005, ApJ, 625, 621

Bundy, K., Fukugita, M., Ellis, R. S., Kodama, T., \& Conselice, C. J. 2004, ApJ, 601, L123

Bundy, K., et al. 2006, ApJ, 651, 120

Caldwell, N., Rose, J. A., \& Concannon, K. D. 2003, AJ, 125, 2891

Caldwell, N., Rose, J. A., Franx, M., \& Leonardi, A. J. 1996, AJ, 111, 78

Cassata, P., et al. 2005, MNRAS, 357, 903

Cattaneo, A., Dekel, A., Devriendt, J., Guiderdoni, B., \& Blaizot, J. 2006, MNRAS, 370, 1651

Cimatti, A., Daddi, E., \& Renzini, A. 2006, A\&A, 453, L29

Cole, S., et al. 2001, MNRAS, 326, 255

Conselice, C. J., Bershady, M. A., Dickinson, M., \& Papovich, C. 2003, AJ, 126,1183

Conselice, C. J., Blackburne, J. A., \& Papovich, C. 2005, ApJ, 620, 564

Cooray, A. 2005, MNRAS, 364, 303 2006, MNRAS, 365, 842

Couch, W. J., \& Sharples, R. M. 1987, MNRAS, 229, 423

Cowie, L. L., Songaila, A., Hu, E. M., \& Cohen, J. G. 1996, AJ, 112, 839

Cox, T. J., Di Matteo, T., Hernquist, L., Hopkins, P. F., Robertson, B., \& Springel, V. 2006a, ApJ, 643, 692
Cox, T. J., Dutta, S. N., Di Matteo, T., Hernquist, L., Hopkins, P. F., Robertson, B., \& Springel, V. 2006b, ApJ, 650, 791

Croom, S. M., Shanks, T., Boyle, B. J., Smith, R. J., Miller, L., Loaring, N. S., \& Hoyle, F. 2001, MNRAS, 325, 483

Croom, S. M., Smith, R. J., Boyle, B. J., Shanks, T., Miller, L., Outram, P. J., \& Loaring, N. S. 2004, MNRAS, 349, 1397

Croom, S. M., et al. 2005, MNRAS, 356, 415

Croton, D. J., et al. 2006, MNRAS, 365, 11

Dasyra, K. M., et al. 2006, ApJ, 638, 745

Dekel, A., \& Birnboim, Y. 2006, MNRAS, 368, 2

De Lucia, G., \& Blaizot, J. 2007, MNRAS, 375, 2

De Propris, R., Liske, J., Driver, S. P., Allen, P. D., \& Cross, N. J. G. 2005, AJ, 130,1516

Di Matteo, T., Springel, V., \& Hernquist, L. 2005, Nature, 433, 604

di Serego Alighieri, S., et al. 2005, A\&A, 442, 125

Dressler, A., \& Gunn, J. E. 1983, ApJ, 270, 7

Driver, S. P., Liske, J., Cross, N. J. G., De Propris, R., \& Allen, P. D. 2005, MNRAS, 360, 81

Drory, N., Bender, R., Feulner, G., Hopp, U., Maraston, C., Snigula, J., \& Hill, G. J. 2004, ApJ, 608, 742

Eisenstein, D. J., \& Hu, W. 1999, ApJ, 511, 5

Elvis, M., et al. 1994, ApJS, 95, 1

Faber, S. M., et al. 2005, ApJ, submitted (astro-ph/0506044)

Farrah, D., Geach, J., Fox, M., Serjeant, S., Oliver, S., Verma, A., Kaviani, A., \& Rowan-Robinson, M. 2004, MNRAS, 349, 518

Farrah, D., et al. 2006, ApJ, 641, L17

Ferrarese, L., \& Merritt, D. 2000, ApJ, 539, L9

Feulner, G., Bender, R., Drory, N., Hopp, U., Snigula, J., \& Hill, G. J. 2003, MNRAS, 342, 605

Floyd, D. J. E., Kukula, M. J., Dunlop, J. S., McLure, R. J., Miller, L., Percival, W. J., Baum, S. A., \& O’Dea, C. P. 2004, MNRAS, 355, 196

Fontana, A., et al. 2004, A\&A, 424, 23 2006, A\&A, 459, 745

Franceschini, A., et al. 2006, A\&A, 453, 397

Franx, M. 1993, ApJ, 407, L5

Gallazzi, A., Charlot, S., Brinchmann, J., \& White, S. D. M. 2006, MNRAS, 370,1106

Gao, L., Springel, V., \& White, S. D. M. 2005, MNRAS, 363, L66

Gebhardt, K., et al. 2000, ApJ, 539, L13

Goto, T. 2005, MNRAS, 357, 937

Graham, A. W., Erwin, P., Caon, N., \& Trujillo, I. 2001, ApJ, 563, L11

Granato, G. L., De Zotti, G., Silva, L., Bressan, A., \& Danese, L. 2004, ApJ, 600,580

Haiman, Z., \& Hui, L. 2001, ApJ, 547, 27

Hasinger, G., Miyaji, T., \& Schmidt, M. 2005, A\&A, 441, 417

Hennawi, J. F., et al. 2006, AJ, 131, 1

Ho, L. C. 2002, ApJ, 564, 120

Hopkins, P. F., \& Hernquist, L. 2006, ApJS, 166, 1

Hopkins, P. F., Hernquist, L., Cox, T. J., Robertson, B., Di Matteo, T., Martini, P., \& Springel, V. 2005a, ApJ, 630, 705

Hopkins, P. F., Hernquist, L., Cox, T. J., Robertson, B., Di Matteo, T., \& Springel, V. 2005b, ApJ, 630, 716

. 2005c, ApJ, 632, 81

2006a, ApJ, 639, 700 2006b, ApJS, 163, 50

Hopkins, P. F., Hernquist, L., Cox, T. J., Robertson, B., \& Springel, V. 2006c, ApJS, 163, 50

Hopkins, P. F., Hernquist, L., Martini, P., Cox, T. J., Robertson, B., Di Matteo, T., \& Springel, V. 2005d, ApJ, 625, L71 
Hopkins, P. F., Narayan, R., \& Hernquist, L. 2006d, ApJ, 643, 641

Hopkins, P. F., Richards, G. T., \& Hernquist, L. 2007, ApJ, 654, 731

Hopkins, P. F., Somerville, R. S., Hernquist, L., Cox, T. J., Robertson, B., \& Li, Y. 2006e, ApJ, 652, 864

Kauffmann, G., \& Haehnelt, M. G. 2000, MNRAS, 311, 576 2002, MNRAS, 332, 529

Kauffmann, G., et al. 2003, MNRAS, 341, 54

Kelson, D. D., Illingworth, G. D., van Dokkum, P. G., \& Franx, M. 2000, ApJ, 531, 184

Kereš, D., Katz, N., Weinberg, D. H., \& Davé, R. 2005, MNRAS, 363, 2

Kollmeier, J. A., et al. 2006, ApJ, 648, 128

Körding, E. G., Jester, S., \& Fender, R. 2006, MNRAS, 372, 1366

Lapi, A., Shankar, F., Mao, J., Granato, G. L., Silva, L., De Zotti, G., \& Danese, L. 2006, ApJ, 650, 42

Le Fèvre, O., et al. 2000, MNRAS, 311, 565

Lemson, G., \& Kauffmann, G. 1999, MNRAS, 302, 111

Li, C., Kauffmann, G., Jing, Y. P., White, S. D. M., Börner, G., \& Cheng, F. Z. 2006, MNRAS, 368, 21

Lidz, A., Hopkins, P. F., Cox, T. J., Hernquist, L., \& Robertson, B. 2006, ApJ, 641,41

Lin, L., et al. 2004, ApJ, 617, L9

Lotz, J. M., Madau, P., Giavalisco, M., Primack, J., \& Ferguson, H. C. 2006a, ApJ, 636, 592

Lotz, J. M., Primack, J., \& Madau, P. 2004, AJ, 128, 163

Lotz, J. M., et al. 2006b, ApJ, submitted (astro-ph/0602088)

Madgwick, D., et al. 2003, ApJ, 599, 997

Magorrian, J., et al. 1998, AJ, 115, 2285

Mandelbaum, R., Seljak, U., Kauffmann, G., Hirata, C. M., \& Brinkmann, J. 2006, MNRAS, 368, 715

Marconi, A., \& Hunt, L. K. 2003, ApJ, 589, L21

Marconi, A., Risaliti, G., Gilli, R., Hunt, L. K., Maiolino, R., \& Salvati, M. 2004, MNRAS, 351, 169

Martini, P. 2004, in Coevolution of Black Holes and Galaxies, ed. L. C. Ho (Cambridge: Cambridge Univ. Press), 169

Martini, P., \& Weinberg, D. H. 2001, ApJ, 547, 12

Masjedi, M., et al. 2006, ApJ, 644, 54

McLure, R. J., \& Dunlop, J. S. 2004, MNRAS, 352, 1390

Menci, N., Fontana, A., Giallongo, E., Grazian, A., \& Salimbeni, S. 2006, ApJ, 647, 753

Meneux, B., et al. 2006, A\&A, 452, 387

Mihos, J. C., \& Hernquist, L. 1994, ApJ, 431, L9 1996, ApJ, 464, 641

Mo, H. J., \& White, S. D. M. 1996, MNRAS, 282, 347

Monaco, P., \& Fontanot, F. 2005, MNRAS, 359, 283

Myers, A. D., et al. 2006, ApJ, 638, 622

Neistein, E., van den Bosch, F. C., \& Dekel, A. 2006, MNRAS, 372, 933

Nelan, J. E., Smith, R. J., Hudson, M. J., Wegner, G. A., Lucey, J. R., Moore, S. A. W., Quinney, S. J., \& Suntzeff, N. B. 2005, ApJ, 632, 137

Norberg, P., et al. 2002, MNRAS, 332, 827

Norton, S. A., Gebhardt, K., Zabludoff, A. I., \& Zaritsky, D. 2001, ApJ, 557, 150

Pannella, M., Hopp, U., Saglia, R. P., Bender, R., Drory, N., Salvato, M., Gabasch, A., \& Feulner, G. 2006, ApJ, 639, L1

Papovich, C., et al. 2006, ApJ, 640, 92

Patton, D. R., et al. 2002, ApJ, 565, 208

Peng, C. Y., Impey, C. D., Rix, H.-W., Kochanek, C. S., Keeton, C. R., Falco, E. E., Lehár, J., \& McLeod, B. A. 2006, ApJ, 649, 616

Percival, W. J., Scott, D., Peacock, J. A., \& Dunlop, J. S. 2003, MNRAS, 338, L31

Pollo, A., et al. 2006, A\&A, 451, 409

Pozzetti, L., et al. 2003, A\&A, 402, 837

Quintero, A. D., et al. 2004, ApJ, 602, 190

Rees, M. J., \& Ostriker, J. P. 1977, MNRAS, 179, 541

Renzini, A. 2006, ARA\&A, 44, 141

Richards, G. T., et al. 2005, MNRAS, 360, 839

-. 2006a, AJ, 131, 2766

2006b, ApJS, 166, 470
Robertson, B., Cox, T. J., Hernquist, L., Franx, M., Hopkins, P. F., Martini, P., \& Springel, V. 2006a, ApJ, 641, 21

Robertson, B., Hernquist, L., Cox, T. J., Di Matteo, T., Hopkins, P. F., Martini, P., \& Springel, V. 2006b, ApJ, 641, 90

Rothberg, B., \& Joseph, R. D. 2004, AJ, 128, 2098

. 2006, AJ, 131, 185

Salpeter, E. E. 1955, ApJ, 121, 161

Sanders, D. B., Soifer, B. T., Elias, J. H., Neugebauer, G., \& Matthews, K. 1988, ApJ, 328, L35

Scannapieco, E., \& Oh, S. P. 2004, ApJ, 608, 62

Scannapieco, E., Silk, J., \& Bouwens, R. 2005, ApJ, 635, L13

Schweizer, F. 1996, AJ, 111, 109

Serber, W., Bahcall, N., Ménard, B., \& Richards, G. 2006, ApJ, 643, 68

Shankar, F., Lapi, A., Salucci, P., De Zotti, G., \& Danese, L. 2006, ApJ, 643, 14

Shepherd, C. W., Carlberg, R. G., Yee, H. K. C., Morris, S. L., Lin, H., Sawicki, M., Hall, P. B., \& Patton, D. R. 2001, ApJ, 560, 72

Sheth, R. K., Mo, H. J., \& Tormen, G. 2001, MNRAS, 323, 1

Shields, G. A., Gebhardt, K., Salviander, S., Wills, B. J., Xie, B., Brotherton,

M. S., Yuan, J., \& Dietrich, M. 2003, ApJ, 583, 124

Silk, J., \& Rees, M. J. 1998, A\&A, 331, L1

Somerville, R. S., Lee, K., Ferguson, H. C., Gardner, J. P., Moustakas, L. A., \& Giavalisco, M. 2004a, ApJ, 600, L171

Somerville, R. S., Primack, J. R., \& Faber, S. M. 2001, MNRAS, 320, 504

Somerville, R. S., et al. 2004b, ApJ, 600, L135

Springel, V., Di Matteo, T., \& Hernquist, L. 2005a, ApJ, 620, L79

2005b, MNRAS, 361, 776

Strateva, I., et al. 2001, AJ, 122, 1861

Straughn, A. N., Cohen, S. H., Ryan, R. E., Hathi, N. P., Windhorst, R. A., \& Jansen, R. A. 2006, ApJ, 639, 724

Tacconi, L. J., Genzel, R., Lutz, D., Rigopoulou, D., Baker, A. J., Iserlohe, C., \& Tecza, M. 2002, ApJ, 580, 73

Toledo, H. M. H., Dultzin-Hacyan, D., Gonzalez, J. J., \& Sulentic, J. W. 1999, AJ, 118, 108

Toomre, A. 1977, in Evolution of Galaxies and Stellar Populations, ed. B. M. Tinsley \& R. B. Larson (New Haven: Yale Univ. Obs), 401

Tran, K.-V. H., Franx, M., Illingworth, G., Kelson, D. D., \& van Dokkum, P. 2003, ApJ, 599, 865

Tran, K.-V. H., Franx, M., Illingworth, G. D., van Dokkum, P., Kelson, D. D., \& Magee, D. 2004, ApJ, 609, 683

Treu, T., Ellis, R. S., Liao, T. X., \& van Dokkum, P. G. 2005, ApJ, 622, L5

Ueda, Y., Akiyama, M., Ohta, K., \& Miyaji, T. 2003, ApJ, 598, 886

Vanden Berk, D. E., et al. 2006, AJ, 131, 84

van der Wel, A., Franx, M., van Dokkum, P. G., \& Rix, H.-W. 2004, ApJ, 601, L5

van der Wel, A., Franx, M., van Dokkum, P. G., Rix, H.-W., Illingworth, G. D., \& Rosati, P. 2005, ApJ, 631, 145

van Dokkum, P. G. 2005, AJ, 130, 2647

van Dokkum, P. G., \& Stanford, S. A. 2003, ApJ, 585, 78

Volonteri, M., Haardt, F., \& Madau, P. 2003, ApJ, 582, 559

Volonteri, M., Salvaterra, R., \& Haardt, F. 2006, MNRAS, 373, 121

White, R. L., Helfand, D. J., Becker, R. H., Glikman, E., \& deVries, W. 2007, ApJ, 654, 99

Willmer, C. N. A., et al. 2006, ApJ, 647, 853

Wolf, C., Wisotzki, L., Borch, A., Dye, S., Kleinheinrich, M., \& Meisenheimer, K. 2003, A\&A, 408, 499

Wolf, C., et al. 2005, ApJ, 630, 771

Wyithe, J. S. B., \& Loeb, A. 2003, ApJ, 595, 614

Xu, C. K., Sun, Y. C., \& He, X. T. 2004, ApJ, 603, L73

Yamada, T., et al. 2005, ApJ, 634, 861

Yan, R., Madgwick, D. S., \& White, M. 2003, ApJ, 598, 848

Yang, X., Mo, H. J., Jing, Y. P., \& van den Bosch, F. C. 2005, MNRAS, 358, 217

Yang, X., Mo, H. J., \& van den Bosch, F. C. 2003, MNRAS, 339, 1057

Zabludoff, A. I., Zaritsky, D., Lin, H., Tucker, D., Hashimoto, Y., Shectman, S. A.,

Oemler, A., \& Kirshner, R. P. 1996, ApJ, 466, 104

Zheng, Z., et al. 2005, ApJ, 633, 791

Zucca, E., et al. 2006, A\&A, 455, 879 